\title{
A Proposal for the Genetic Lateral Tuning of Linguistic Fuzzy Systems and Its Interaction With Rule Selection
}

\author{
Rafael Alcalá, Jesús Alcalá-Fdez, and Francisco Herrera
}

\begin{abstract}
Linguistic fuzzy modeling allows us to deal with the modeling of systems by building a linguistic model which is clearly interpretable by human beings. However, since the accuracy and the interpretability of the obtained model are contradictory properties, the necessity of improving the accuracy of the linguistic model arises when complex systems are modeled. To solve this problem, one of the research lines in recent years has led to the objective of giving more accuracy to linguistic fuzzy modeling without losing the interpretability to a high level.

In this paper, a new postprocessing approach is proposed to perform an evolutionary lateral tuning of membership functions, with the main aim of obtaining linguistic models with higher levels of accuracy while maintaining good interpretability.

To do so, we consider a new rule representation scheme base on the linguistic 2-tuples representation model which allows the lateral variation of the involved labels. Furthermore, the cooperation of the lateral tuning together with fuzzy rule reduction mechanisms is studied in this paper, presenting results on different real applications. The obtained results show the good performance of the proposed approach in high-dimensional problems and its ability to cooperate with methods to remove unnecessary rules.
\end{abstract}

Index Terms-Fuzzy rule-based systems, genetic algorithms, interpretability, linguistic 2 -tuples representation, rule selection, tuning.

\section{INTRODUCTION}

$\mathbf{F}$ UZZY modeling (FM) - i.e., system modeling with fuzzy rule-based systems (FRBSs) - may be considered as an approach used to model a system by making use of a descriptive language based on fuzzy logic [1], [2] with fuzzy predicates [3]. Several types of modeling can be performed depending on the desired degree of interpretability and accuracy of the final model. Unfortunately, both requirements are contradictory properties directly depending on the learning process and model structure.

In this framework, one of the most important areas is the linguistic FM, where the interpretability of the obtained model is the main requirement. This task is usually developed by means of linguistic FRBSs (also called Mamdani FRBSs [4],

Manuscript received January 18, 2005; revised May 3, 2006 and July 5, 2006. This work was supported by the Spanish Ministry of Science and Technology under Projects TIC-2002-04036-C05-01 and TIN-2005-08386-C05-01.

The authors are with the Department of Computer Science and Artificial Intelligence, University of Granada, 18071 Granada, Spain (e-mail: alcala@ decsai. ugr.es; jalcala@decsai.ugr.es; herrera@decsai.ugr.es).

Color versions of one or more of the figures in this paper are available online at http://ieeexplore.iee.org.

Digital Object Identifier 10.1109/TFUZZ.2006.889880
[5]), which use fuzzy rules composed of linguistic variables [3] taking values in a term set with a real-world meaning. One of the problems associated with the linguistic FM is its lack of accuracy when modeling some complex systems. This is due to the inflexibility of the concept of the linguistic variable, which imposes hard restrictions on the fuzzy rule structure [6]. This drawback leads linguistic FM sometimes to move away from the desired tradeoff between interpretability and accuracy, thus losing the usefulness of the model. To overcome this problem, many different possibilities to improve the accuracy of linguistic FM while preserving its intrinsic interpretability have been considered in the specialized literature [7].

One of the most widely used approaches to improving the performance of FRBSs, known as tuning, consists of refining a previous definition of the data base (DB) once the rule base (RB) has been obtained. Generally, tuning is a variation in the shape of the membership functions (MFs) that improves their global interaction with the main aim of inducing better cooperation among the rules [8]-[12]. In this way, the real aim of the tuning is to find the best global configuration of the MFs and not only to find independently specific MFs.

In the framework of linguistic FM and fuzzy control, there is a predominant use of triangular shaped MFs because of their simplicity with more or less similar results with respect to other approaches [13], [14] (trapezoidal or Gaussian). Classically, the tuning methods refine the three definition parameters that identify the triangular MFs associated to the labels comprising the DB [9], [13]. These three parameters are also dependent among themselves for each MF. In the case of problems with many variables, these two facts, the dependency among MFs and the dependency among the three definition points, lead to tuning models handling very complex search spaces which affect the good performance of the optimization methods.

In this paper, we present a new FRBS postprocessing approach to perform an evolutionary lateral tuning of the linguistic variables. It uses a new rule representation model based on the linguistic 2-tuples representation [15] that allows the lateral displacement of the labels by considering only one parameter (slight displacements to the left/right of the original MFs). This way to work involves a simplification of the search space that eases the derivation of optimal models and therefore improves the desired tradeoff, especially in complex or high-dimensional problems.

In addition, rule selection methods directly aggregate multiple rules and/or select a subset of rules from a given fuzzy rule set in order to minimize the number of rules [16], [17]. 


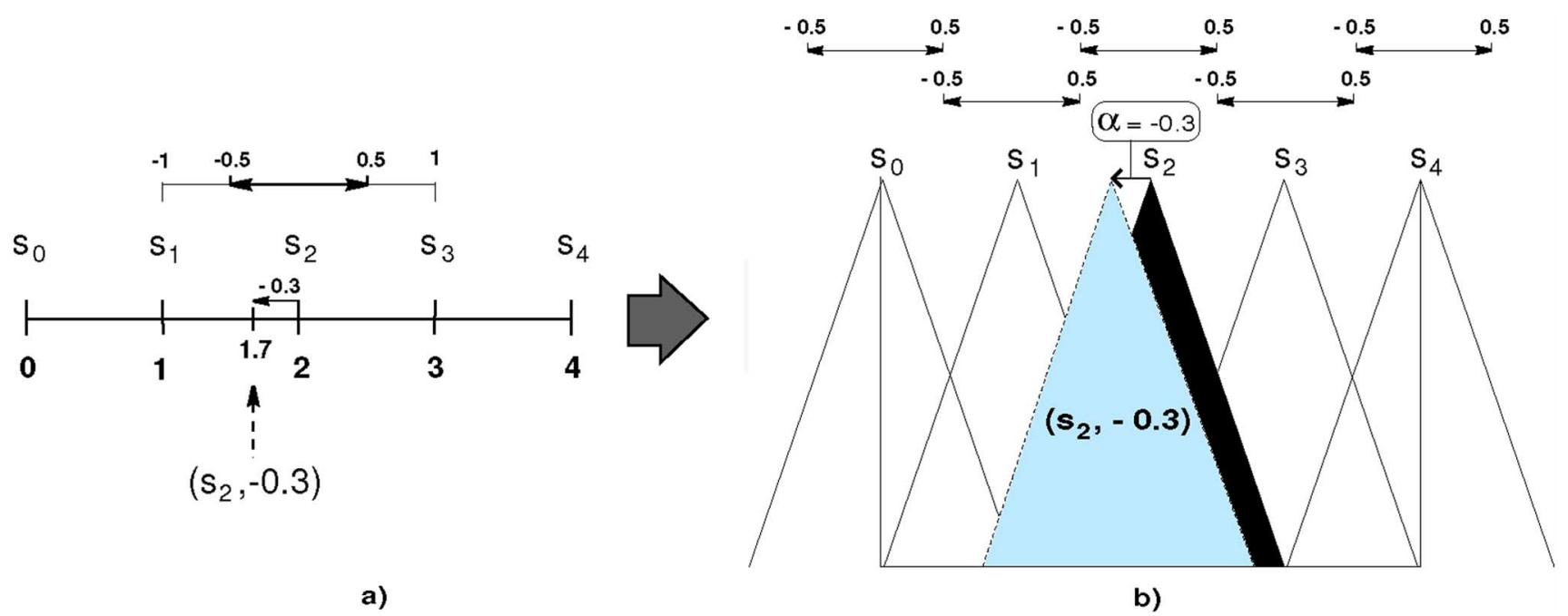

Fig. 1. Symbolic translation of a linguistic label and lateral displacement of the involved MF.

The combination of lateral tuning with rule selection methods can present a positive synergy, reducing the tuning search space, easing system readability and even improving system accuracy. In this paper, the cooperation between lateral tuning and a mechanism for rule selection is also studied, showing their performance on different applications.

This paper is arranged as follows. The next section presents the proposed lateral tuning, the linguistic rule representation model (based on the linguistic 2-tuples), and the main differences with respect to the classic approach. Section III details the evolutionary method proposed to perform the lateral tuning of FRBSs. In Section IV, the cooperation between the lateral tuning and a rule selection mechanism is proposed, presenting the evolutionary method to perform both together. Section V shows an experimental study of the performance of the method applied to FRBSs obtained from automatic learning methods and from expert knowledge, respectively, considering two real-world electrical distribution problems (small and medium complexity) and a highly complex real-world problem for the fuzzy control of a heating, ventilating, and air conditioning (HVAC) system. Section VI gives some concluding remarks. The Appendix describes the problem of fuzzy control of HVAC systems.

\section{A Proposal for the Lateral Tuning of FuZZY RULE-BASED SYSTEMS}

In this section, we introduce our proposal for the lateral tuning of fuzzy systems, presenting the rule structure and two different tuning approaches (global approach and local approach). Then, the main differences between the lateral and the classic tuning of triangular MFs are discussed.

\section{A. Lateral Tuning}

The main aim of this paper is to reduce the tuning search space by proposing a new tuning model of FRBSs based on the linguistic 2-tuples representation scheme introduced in [15] that allows the lateral displacement of the support of a label maintaining the interpretability associated with the final linguistic model at a reasonable level. In [15], the lateral displacement represented by a linguistic 2-tuple is named symbolic translation of a label.

The symbolic translation of a label is a number within the interval $[-0.5,0.5)$, expressing with this interval the domain of a label when it is moving between its two adjacent lateral labels [see Fig. 1(a)]. Let us consider a set of labels $S$ representing a fuzzy partition. Formally, to represent the symbolic translation of a label in $S$ we have the 2-tuple

$$
\left(s_{i}, \alpha_{i}\right), s_{i} \in S, \alpha_{i} \in[-0.5,0.5) .
$$

In fact, the symbolic translation of a label involves the lateral displacement of its associated MF. As an example, Fig. 1 shows the symbolic translation of a label represented by the pair $\left(s_{2},-0.3\right)$ together with the lateral displacement of the corresponding MF. Both the linguistic 2-tuples representation model and the elements needed for linguistic information comparison and aggregation are presented and applied to the decision-making framework in [15].

In the context of the FRBSs, the linguistic 2-tuples could be used to represent the MFs comprising the linguistic rules. This introduces a new model for rule representation that allows the tuning of the MFs by learning their respective lateral displacements. Next, we present this approach by considering a simple control problem.

Let us consider a control problem with two input variables, one output variable, and a DB defined from experts determining the MFs for the following labels:

$$
\begin{aligned}
\text { Error } \rightarrow\{N, Z, P\}, \quad \nabla \text { Error } \rightarrow\{N, Z, P\} \\
\text { Power } \rightarrow\{L, M, H\} .
\end{aligned}
$$

Based on this DB definition, Fig. 2 shows the concept of classic rule and linguistic 2-tuples represented rule. Analyzed from point of view of rule interpretability, we could interpret the 2-tuples represented rule (i.e., a tuned rule) as follows.

If the Error is "higher than Zero" and the Error Variation is "a little smaller than Positive" then the Power is "a bit smaller than High." 


\section{Classic Rule:}

R1: If the Error is Zero and the Error Variation is Positive then the Power is High

Rule with 2-tuples Representation:

R1: If the Error is (Zero, 0.3) and the Error Variation is (Positive, -0.2) then the Power is (High, -0.1)

Fig. 2. Classic rule and rule with 2-tuple representation.

This proposal decreases the complexity of the tuning problem, since the three parameters considered per label are reduced to only one symbolic translation parameter. As to how to perform the lateral tuning, there are two different possibilities, one more interpretable and one more accurate.

- Global Tuning of the Semantics: In this case, the tuning is applied to the level of linguistic partition. In this way, the pair $\left(X_{i}\right.$, label) takes the same tuning value in all the rules where it is considered. For example, " $X_{i}$ is (High, 0.3)" will present the same value for those rules in which the couple " $X_{i}$ is High" is initially considered. That is, only one displacement parameter is considered for each label on the DB.

Considering this approach, the global interpretability of the final FRBS is maintained. It could be compared to the classic tuning of the DB considering descriptive fuzzy rules [18], i.e., a global collection of fuzzy sets is considered by all the fuzzy rules.

- Local Tuning of the Rules: In this case, the tuning is applied at rule level. The pair $\left(X_{i}\right.$, label) is tuned in a different way in each rule, based on the quality measures associated to the tuning method (usually the system error). That is, different displacement parameters are considered for each label on the DB depending on the rule in which this label is considered (one parameter per rule and variable). For example, we could have the pair $\left(X_{i}\right.$, High) in different rules with different displacement parameters.

Rule $k: X_{i}$ is (High, 0.3) (more than high).

Rule $j: X_{i}$ is (High, -0.2) (a little lower than high).

In this case, the global interpretability is lost to some degree and the obtained model should be interpreted from a local point of view. This approach could be compared to the classic tuning of approximate fuzzy rules [18], i.e., each fuzzy rule has associated its own local fuzzy sets. However, in our case, the tuned labels are still related to the initial ones, preserving the global interpretability to some degree. This way to work gives more freedom to find more accurate FRBSs but handles a higher search space than the global approach. Therefore, this approach can obtain more accurate but less interpretable linguistic models than the global approach. The use of a global or a local approach depends on the problem being solved and on the expert needs.

Once both approaches have been presented, some aspects related to the search space reduction and the interpretability of the obtained models should be clarified. Naturally, these aspects are closely related to the type of tuning performed (global or local).
We have pointed out that lateral tuning decreases the tuning problem complexity, since only one symbolic translation parameter is considered per label. We must clarify that this is true individually considering both approaches, global and local. Therefore, global lateral tuning reduces the search space with respect to the classic tuning of the DB (linguistic rules) and local lateral tuning reduces the search space with respect to the classic tuning of approximate fuzzy rules. From the point of view of interpretability, they should be individually compared again, since the global approach tries to obtain more interpretable models and the local approach tries to obtain more accurate ones.

\section{B. Main Differences Between Classic and Lateral Tuning}

As has been mentioned, the classic tuning of MFs usually works with three parameters per MF (triangular shape). The total number of these parameters grows exponentially in terms of the number of variables and the number of labels per variable. Usually, five or seven labels per variable are considered, and never more than nine. However, the number of variables depends on each specific problem, causing the problem complexity to grow exponentially when the number of system variables increases.

This fact involves a problem for the tuning methods. The most well-known approaches are those based on the use of neural networks and those based on the use of genetic algorithms (GAs). In the first case, we could highlight the ANFIS method [11], which needs to represent all the possible combinations of rules in the second layer and provokes a memory overflow when there are more than four or five input variables. Considering GAs, a chromosome with a large number of related genes is necessary to represent all the involved parameters, and this fact leads to convergence problems of the evolutionary methods [19], [20].

The main difference between lateral tuning and the classic approach is the reduction of the search space, focusing the search only on the MF support position. Although lateral tuning has less freedom than the classic approach, the reduction of the search space could lead to improved performance of the tuning method, especially in complex or highly multidimensional problems, since this allows us to obtain easily the best global interaction between the MFs, thereby ensuring a good covering degree of the input data.

Let us consider a simplification of the tuning problem to calculate the probability of a solution (chromosome) to move in the direction of the global optimum. Fig. 3 shows the probability of an MF to move in the direction of the global optimum for both approaches (probability of moving the definition points in the successful direction). Therefore, the probability $P$ of obtaining a better global combination of MFs (with $m$ being the number of total labels) in the case of lateral tuning $\left(P_{\text {Lat }}\right)$ and classic tuning $\left(P_{\mathrm{Cl}}\right)$ is

$$
P=\prod_{i=1}^{m} P_{i} \text {, i.e. } P_{\mathrm{Lat}}=0.5^{m} \text { and } P_{\mathrm{Cl}}=0.5^{3 * m} \text {. }
$$

For example, in a problem with five variables, five labels per variable, and considering the global tuning approach, we have 
With lateral displacement

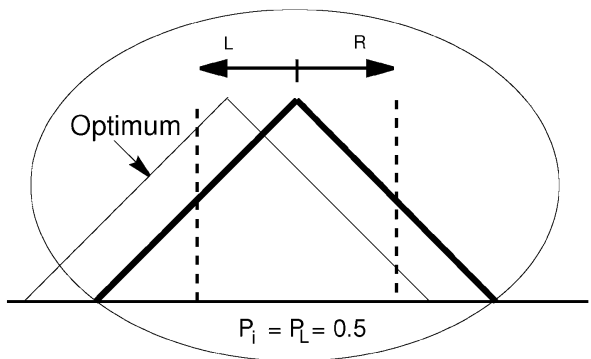

Considering the three definition points

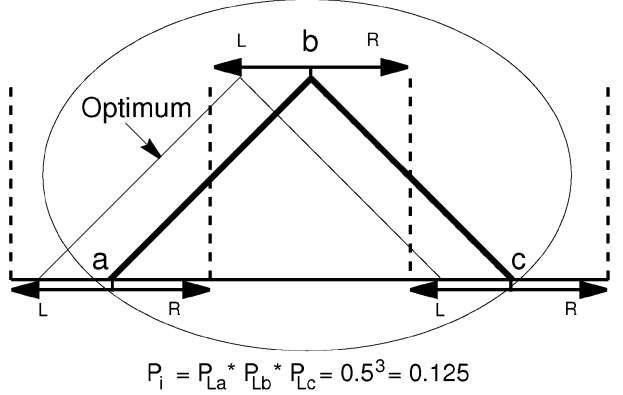

Fig. 3. Probability of an MF to move to the optimum.

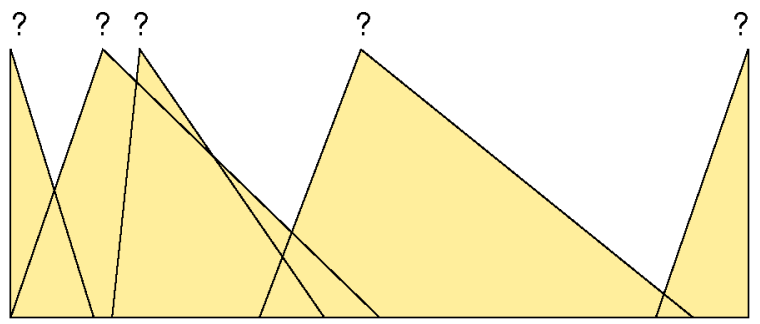

Fig. 4. Tuned fuzzy partition and its loss of interpretability.

$P_{\text {Lat }} \approx 3 \cdot 10^{-8}$ and $P_{\mathrm{Cl}} \approx 3 \cdot 10^{-23}$, which are quite different. This is a simplification of the problem because the tuning process also depends on factors such as the rules that establish the relations between the MFs, the crossover and mutation operators, etc. However, this simple example can give us an idea of the differences between both approaches.

On the other hand, classic tuning could not maintain a high global degree of interpretability. Therefore, it is important to point out that the design process could lead to complex fuzzy partitions in the DB, which could make interpretation of the system by an expert difficult. Fig. 4 shows an example of MFs with complex shapes, which lose the global interpretability from the point of view of the linguistic FM.

To determine if a specific DB is interpretable or not is a subjective and difficult task. In any case, some researchers have studied this issue proposing several properties in order to ensure a good interpretability through the MF optimization process [21]-[23]. Several constraints can be applied in the design process in order to obtain a DB considering one or more of these properties and maintaining the linguistic model comprehensibility at the highest possible level [18], [24]-[26].

Indeed, the necessary constraints to ensure the semantic integrity of the DB make the derivation process less flexible, but they maintain the interpretability of the model as well as reduce the risk of overfitting the final FRBS. Therefore, it is very important to apply these constraints in an intelligent way, maintaining the tuning process ability/potentiality to improve the system performance ass much as possible [27].

In the case of lateral tuning, the 2-tuples represented labels can be interpreted with respect to the initial ones (see the 2-tuples represented rule interpretation in the previous subsection). However, since we can obtain the corresponding triangular MFs from the parameters $\alpha$ applied to each label, an FRBS based on linguistic 2-tuples could be also represented as a classic Mamdani FRBS [4], [5]. In this way, from the point of view of interpretability:

- the original shapes of the MFs are maintained (in our case triangular and symmetrical), by laterally changing the location of their supports;

- the lateral variation of the involved labels is restricted to a short interval, ensuring overlapping between two adjacent labels to some degree but preventing their vertex points from crossing.

\section{EVOlutionary POSTPROCESSING METHODS FOR LATERAL} TUNING

The automatic definition of fuzzy systems can be considered as an optimization or search process and nowadays, evolutionary algorithms, particularly GAs [19], [20], are considered as the most well-known and used global search technique. Moreover, the genetic coding that they use allows them to include prior knowledge and to use it for leading the search. For this reason, evolutionary algorithms have been successfully applied to learn fuzzy systems in recent years, giving way to the appearance of so-called genetic fuzzy systems [9], [28].

Evolutionary algorithms in general and GAs in particular have been widely used in the tuning of FRBSs. In this paper, we consider two different evolutionary models that will be used in our experimental study: the genetic model of CHC [29] and a steady-state GA [30]. Each one of these approaches is motivated by the kind of problem being solved (some comments on both approaches are presented at the end of the next subsection). At this point, we must remark that no mutation is considered in CHC (imposed by the evolutionary model) and that no mutation is considered in the steady-state approach in order to improve the algorithm convergence in problems that must be solved with a small number of evaluations.

Next, we present the main components needed to design the evolutionary tuning algorithms. Then, a total of four algorithms will be proposed to perform the global and the local lateral tuning following both evolutionary models ( $\mathrm{CHC}$ or steadystate, depending on the type of problem being solved).

\section{A. Main Components of Evolutionary Algorithms}

The main components needed to design the evolutionary tuning algorithms are:

- coding scheme and initial gene pool;

- chromosome evaluation;

- crossover operator;

- restarting approach;

- evolutionary model. 
1) Coding Scheme and Initial Gene Pool: Taking into account that two different types of tuning have been proposed (global tuning of the semantics and local tuning of the rules), there are two different kinds of coding schemes. In both cases, a real coding is considered, i.e., the real parameters are the GA representation units (genes). In the following both schemes are presented.

- Global Tuning of Semantics: joining of the parameters of the fuzzy partitions. Let us consider the following number of labels per variable: $\left(m^{1}, m^{2}, \ldots, m^{n}\right)$, with $n$ being the number of variables. Then, a chromosome has the form (where each gene is associated to the lateral displacement of the corresponding label in the DB)

$$
C_{T}=\left(c_{11}, \ldots, c_{1 m^{1}}, c_{21}, \ldots, c_{2 m^{2}}, \ldots, c_{n 1}, \ldots, c_{n m^{n}}\right) .
$$

See the $C_{T}$ part of Fig. 8(a) (in the next section) for an example of coding scheme considering this approach.

- Local Tuning of the Rules: joining of the rule parameters. Let us consider that the FRBS has $M$ rules, $(R 1, R 2, \ldots, R M)$, with $n$ system variables. Then, the chromosome structure has the following form (where each gene is associated to the lateral displacement of the corresponding label for each rule):

$C_{T}=\left(c_{11}, \ldots, c_{1 n}, c_{21}, \ldots, c_{2 n}, \ldots, c_{M 1}, \ldots, c_{M n}\right)$

See the $C_{T}$ part of Fig. 8(b) (in the next section) for an example of a coding scheme considering this approach.

To make use of the available information, the initial FRBS obtained from automatic fuzzy rule learning methods or from expert knowledge is included in the population as an initial solution. To do so, the initial pool is obtained with the first individual having all genes with value 0.0 and the remaining individuals generated at random in $[-0.5,0.5)$.

2) Chromosome Evaluation: The chromosome evaluation will depend on the kind of system being modeled.

- When a set of input-output training data representing the system operation is available (data-driven learning), to evaluate a determined chromosome we will use the well-known mean square error (MSE):

$$
\mathrm{MSE}=\frac{1}{2 \cdot N} \sum_{l=1}^{N}\left(F\left(x^{l}\right)-y^{l}\right)^{2}
$$

where $N$ is the data set size, $F\left(x^{l}\right)$ the output obtained from the FRBS decoded from the said chromosome when the $l$ th example is considered, and $y^{l}$ the known desired output.

- When the initial FRBS is obtained from expert knowledge and/or a validated model of the real system is available, each chromosome is evaluated by means of a system simulation that measures the FRBS performance. This approach is usually effective in control problems.

3) Crossover Operator: Two different crossover operators are applied depending on the evolutionary model considered, $\mathrm{CHC}$ or the steady-state GA. They are based on the concept of

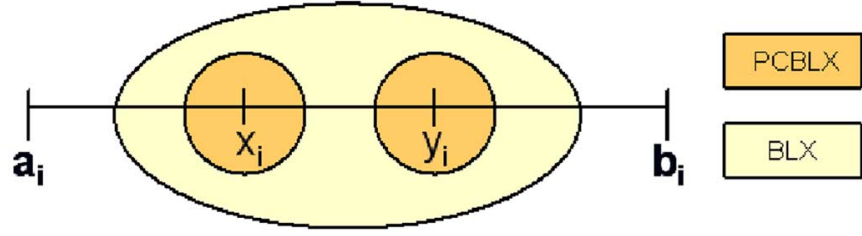

Fig. 5. Diagram of the performance of the crossover operators based on environments.

environments (the offspring are generated around their parents). These kinds of operators present good cooperation when they are introduced within evolutionary models forcing the convergence by pressure on the offspring (as the case of $\mathrm{CHC}$ or the steady-state approach). Particularly, we consider the parent-centric BLX (PCBLX) operator [31] and the BLX- $\alpha$ combined with an arithmetic-based crossover. Fig. 5 shows the performance of these kinds of operators, which allow the offspring genes to be around the genes of one parent (PCBLX) or around a wide zone determined by both parent genes (BLX).

These crossover operators are described as follows. Let us assume $X=\left(x_{1} \cdots x_{g}\right)$ and $Y=\left(y_{1} \cdots y_{g}\right),\left(x_{i}, y_{i} \in\left[a_{i}, b_{i}\right] \subset\right.$ $\Re, i=1 \cdots g)$, are two real-coded chromosomes that are going to be crossed.

- CHC approach: In this case, we consider the PCBLX operator [31], which is based on BLX- $\alpha$. The PCBLX operator generates the following two offspring:

1) $Z=\left(z_{1} \cdots z_{g}\right)$, where $z_{i}$ is a randomly (uniformly) chosen number from the interval $\left[l_{i}^{z}, u_{i}^{z}\right.$, with $l_{i}^{z}=$ $\max \left\{a_{i}, x_{i}-I_{i}\right\}, u_{i}^{z}=\min \left\{b_{i}, x_{i}+I_{i}\right\}$, and $I_{i}=$ $\left|x_{i}-y_{i}\right|$

2) $V=\left(v_{1} \cdots v_{g}\right)$, where $v_{i}$ is a randomly (uniformly) chosen number from the interval $\left[l_{i}^{v}, u_{i}^{v}\right]$, with $l_{i}^{v}=$ $\max \left\{a_{i}, y_{i}-I_{i}\right\}$ and $u_{i}^{v}=\min \left\{b_{i}, y_{i}+I_{i}\right\}$.

- Steady-state approach: A BLX- $\alpha$ crossover [31] and a hybrid between a BLX- $\alpha$ and an arithmetical crossover [32] are considered.

1) Using the BLX- $\alpha$ crossover [31] (with $\alpha=0.3$ ), one descendent $Z=\left(z_{1}, \cdots, z_{g}\right)$ is obtained, where $z_{i}$ is randomly (uniformly) generated within the interval $\left[l_{i}, u_{i}\right]$, with $l_{i}=\max \left\{a_{i}, c_{\min }-I\right\}, u_{i}=$ $\min \left\{b_{i}, c_{\max }+I\right\}, c_{\min }=\min \left\{x_{i}, y_{i}\right\}, c_{\max }=$ $\max \left\{x_{i}, y_{i}\right\}$ and $I=\left(c_{\max }-c_{\min }\right) \cdot \alpha$.

2) The application of an arithmetical crossover [32] in the wider interval considered by BLX- $\alpha,\left[l_{i}, u_{i}\right]$ results in the next descendent

$$
V \text { with } v_{i}=a \cdot l_{i}+(1-a) \cdot u_{i}
$$

where $a \in[0,1]$ is a random parameter generated each time this crossover operator is applied. In this way, this operator performs the same gradual adaptation in each gene, which involves a faster convergence in the algorithm.

4) Restarting Approach: To get away from local optima, this algorithm uses a restart approach [29]. In this case, the best chromosome is maintained and the remaining are generated at random within the corresponding variation intervals $[-0.5,0.5)$. 
It follows the principles of $\mathrm{CHC}$ [29], performing the restart procedure when a threshold value is reached or all the individuals coexisting in the population are very similar.

5) Evolutionary Model: We will consider two different evolutionary models depending on the need to force premature convergence. Sometimes, a long computational time is required to evaluate a chromosome, e.g., when we only have the real system or a system simulation taking a long time per evaluation. In these cases, we only have a small number of evaluations (e.g., no more than 5000) and a steady-state GA will be considered to provoke fast convergence. On the other hand, when there are no time restrictions, we will consider a population-based selection approach, by using the $\mathrm{CHC}$ evolutionary model in order to perform a better global search. They are used in the following way.

- The Genetic Model of CHC [29]: This makes use of a "population-based selection" approach. $N$ parents and their corresponding offspring are combined to select the best $N$ individuals to take part of the next population. The $\mathrm{CHC}$ approach makes use of an incest prevention mechanism and a restarting process to provoke diversity in the population, instead of the well-known mutation operator. This incest prevention mechanism will be considered in order to apply the PCBLX operator, i.e., two parents are crossed if their hamming distance divided by two is over a predetermined threshold $L$. Since we consider a real coding scheme, we have to transform each gene considering a gray code with a fixed number of bits per gene (BITSGENE) determined by the system expert. In this way, the threshold value is initialized as

$$
L=\left(\# \text { Genes } C_{T} *\right. \text { BITSGENE)/4.0. }
$$

Following the original CHC scheme, $L$ is decreased by one when the population does not change in one generation. In order to avoid very slow convergence, in our case, $L$ will be also decreased by one when no improvement is achieved with respect to the best chromosome of the previous generation. The algorithm restarts when $L$ is below zero.

- The Steady-State Approach [30]: The steady-state GA consists of selecting two of the best individuals in the population and combining them to obtain two offspring. Then, these two new individuals are included in the population by replacing the two worst individuals if the former are better adapted than the latter. An advantage of this technique is that good solutions are used as soon as they are available. Therefore, the convergence is accelerated while the number of evaluations needed is decreased. Furthermore, no mutation will be considered in order to favor the exploitation with respect to the exploration. For this reason, we also consider a restarting approach to avoid local optima. The algorithm restarts when the difference between the worst and the best chromosome fitness values is lesser than $1 \%$ of the initial solution fitness value.

\section{B. Proposed Evolutionary Algorithms}

Considering both evolutionary models, we propose four different algorithms to perform lateral tuning.
1) Generate the initial population with $N$ chromosomes.

2) Evaluate the population (MSE) and initialize the threshold value $L$.

3) Generate the next population:

- Shuffle the population.

- Select the parents two by two. Each pair is crossed (PCBLX) if the hamming distance between the parent Gray codings divided by 2 is over $L$.

- Evaluate the new individuals (MSE).

- Join the parents with their offspring and select the best $N$ individuals to take part of the next population.

4) If the best chromosome does not change, $L=L-1$. And, if there are no new individuals in the population, $L=L-1$.

5) If $L<0$, restart the population.

6) If the maximum number of evaluations is not reached, go to Step 3.

Fig. 6. Data-driven algorithm (CHC approach).

1) Generate the initial population with $N$ chromosomes.

2) Evaluate the population (System Simulation). Let $F_{i n i}$ be the initial solution fitness.

3) Perform a probabilistic selection of two of the best individuals in the population.

4) Cross these individuals (hybrid BLX- $\alpha$ /arithmetical).

5) Evaluate the two offspring (System Simulation).

6) Replace the two worst individuals in the population by the two new individuals if they are better adapted. Let $F_{\text {best }}$ and $F_{\text {worst }}$ be the best and the worst chromosome fitness values.

7) If $\left(F_{\text {worst }}-F_{\text {best }}<0.01 * F_{\text {ini }}\right)$, restart the population.

8) If the maximum number of evaluations is not reached, go to Step 3.

Fig. 7. System simulation-based algorithm (steady-state approach).

1) The first two consider the CHC approach (for data driven problems that allow a reasonable number of evaluations) by following the algorithm described in Fig. 6 with different coding schemes:

- $\mathrm{GL}_{d d}$ : taking into account the global coding scheme presented in Section III-A1 to perform a global lateral tuning of the semantics;

- $\mathrm{LL}_{d d}$ : taking into account the local coding scheme presented in Section III-A1 to perform a local lateral tuning of the rules.

2) Considering the steady-state approach described in Fig. 7 (for problems that allow a small number of evaluations), we also have two different algorithms:

- $\mathrm{GL}_{s s}$ : using the global coding scheme;

- $\mathrm{LL}_{s s}$ : using the local coding scheme.

\section{Interaction Between the Rule Selection and the LATERAL TUNING}

Sometimes, a large number of fuzzy rules must be used to reach an acceptable degree of accuracy. However, an excessive number of rules makes it difficult to understand the model operation. Moreover, we may find different kinds of rules in a large fuzzy rule set: irrelevant rules, which do not contain significant information; redundant rules, whose actions are covered by other rules; erroneous rules, which are incorrectly defined and distort the FRBS performance; and conflicting rules, which perturb the FRBS performance when they coexist with others. 
These kinds of rules are usually obtained in the following situations.

i) When the final RB is generated by only considering the expert's knowledge, redundant, conflicting, and even erroneous rules are usually obtained.

ii) When we consider a fuzzy rule set learning process with a tendency to generate too many rules (sometimes advisedly), redundant or conflicting rules could be found in the obtained RB. For example, methods such as the Wang and Mendel algorithm [33] or the input space oriented strategy [34] are biased by covering criteria trying to ensure a high covering degree that sometimes is not needed. The mixed method (MM) [35] consists of adding rules to the linguistic model obtained by the Wang and Mendel algorithm in the fuzzy input subspaces that have examples but do not yet have a rule, trying to improve the accuracy of the linguistic model by adding even more rules. However, these kinds of methods are still useful to obtain a set of promising candidate rules in order to subsequently select those with the best cooperation as a second stage [36], [37]. In these cases, two main situations favor the generation of these kinds of rules.

- In complicated multidimensional problems with highly nonlinear input-output relations, in which the cooperation of the obtained rules is more difficult to obtain and more rules are usually needed.

- In high-dimensional problems, in which the number of rules in the RB grows exponentially as more inputs are added. A large rule set might contain more redundant or even conflicting rules.

To face this problem, a fuzzy rule set reduction process can be developed to achieve the goal of minimizing the number of rules used while maintaining (or even improving) the FRBS performance. To do that, erroneous and conflicting rules that degrade the performance are eliminated, obtaining a more cooperative fuzzy rule set and therefore involving a potential improvement in the system accuracy. Moreover, in many cases accuracy is not the only requirement of the model but also interpretability becomes an important aspect. Reducing the model complexity is a way to improve the system readability, i.e., a compact system with few rules requires a minor effort to be interpreted.

Fuzzy rule set reduction is generally applied as a postprocessing stage, once an initial fuzzy rule set has been derived. We may distinguish between two different approaches to obtain a compact fuzzy rule set.

1) Selecting Fuzzy Rules: This involves obtaining an optimal subset of fuzzy rules from a previous fuzzy rule set by selecting some of them. We may find several methods for rule selection, with different search algorithms that look for the most successful combination of fuzzy rules [17], [36]-[39]. In [40], an interesting heuristic rule selection procedure is proposed where, by means of statistical measures, a relevance factor is computed for each fuzzy rule in the linguistic FRBSs to subsequently select the most relevant ones. The philosophy of ordering the rules with respect to an importance criterion and selecting a subset of the best seems similar to the orthogonal transformation-methods considered by Takagi-Sugeno-type FRBSs [41], [42].
2) Merging Fuzzy Rules: This is an alternative approach that reduces the fuzzy rule set by merging the existing rules. In [43], the authors propose merging neighboring rules, i.e., fuzzy rules where the linguistic terms used by the same variable in each rule are adjacent. Another proposal is presented in [44], where a special consideration to the merging order is made. In Takagi-Sugeno-type FRBSs, processes that simplify the fuzzy models by merging fuzzy rules have also been proposed [48], [49].

These kinds of techniques for rule reduction could easily be combined with other postprocessing techniques to obtain more compact and accurate FRBSs. In this way, some works have considered the selection of rules together with the tuning of MFs by coding all of them (rules and parameters) in the same chromosome [48], [49].

\section{A. Positive Synergy Between Both Approaches}

There are several reasons explaining the positive synergy between the rule selection and the tuning of MFs. Some of them are as follows.

- The tuning process is affected when erroneous or conflictive rules are included in the initial RB. When the RB of a model being tuned contains bad rules (greatly increasing system error), the tuning process tries to reduce the effect of these kinds of rules, adapting them and the remaining ones to avoid the bad performance of such rules. This way of working imposes strict restrictions, reducing the process ability to obtain precise linguistic models. Furthermore, in some cases this also affects the interpretability of the model, since the MFs comprising bad rules do not have the shape and location that best represents the information being modeled.

This problem grows as the problem complexity grows (i.e., problems with a large number of variables and/or rules) and when the rule generation method does not ensure the generation of rules with good quality (e.g., when the initial RB is obtained from experts). In these cases, the tuning process is very complicated because the search ability is dedicated to reducing the bad performance of some rules instead of improving the performance of the remaining ones. In these cases, rule selection could help the tuning mechanism by removing the rules that really degrade the accuracy of the model.

- Sometimes redundant rules cannot be removed by only using a rule selection method, since these kinds of rules could reinforce the action of poor rules improving the model accuracy. The tuning of MFs can change the performance of these rules, making the reinforcement action unnecessary, and therefore helping the rule selection technique to remove redundant rules.

Therefore, combining rule selection and tuning approaches could result in important improvements in the system accuracy, maintaining the interpretability at an acceptable level [48]-[50]. However, in some cases, the search space considered when both techniques are combined is too large, which could provoke the derivation of suboptimal models [49].

In this section, we propose the selection of a cooperative set of rules from a candidate fuzzy rule set together with the learning 
a)

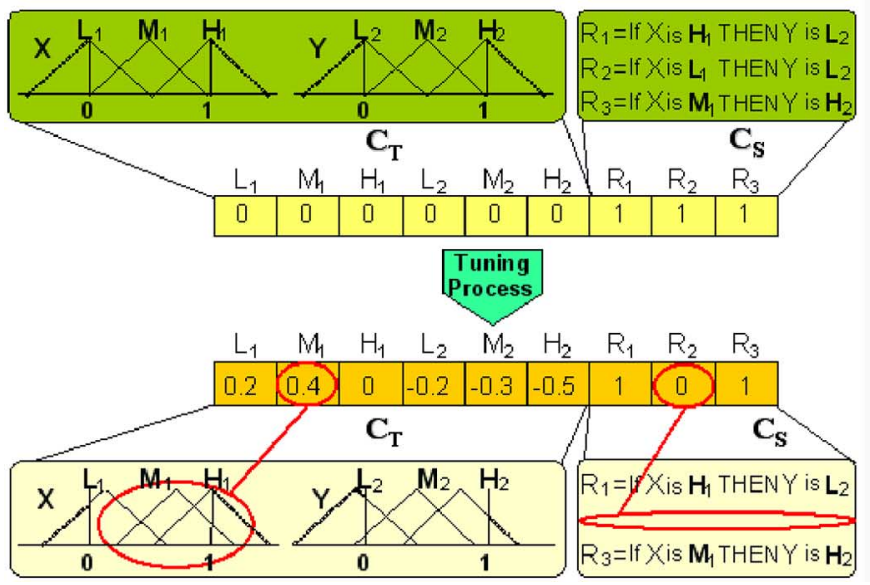

b)

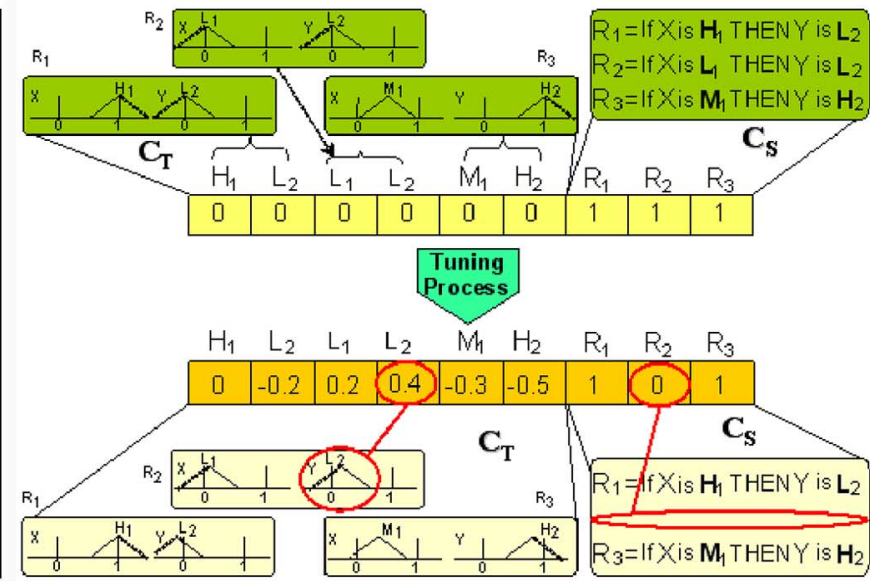

Fig. 8. Example of coding scheme considering the lateral tuning and rule selection: (a) global approach and (b) local approach.

of the symbolic translation parameters. This pursues the following aims:

- to improve the linguistic model accuracy selecting the set of rules best cooperating while lateral tuning is performed to improve the location of the MFs;

- to obtain simpler, and thus easily understandable, linguistic models by removing unnecessary rules;

- to favor the combined action of the tuning and selection strategies (which involves a larger search space) by considering the simpler search space of the lateral tuning (only one parameter per label).

\section{B. Evolutionary Algorithms}

To select the subset of rules that cooperate best and to obtain the lateral translation parameters, we consider a GA that codes all of them (rules and parameters) in one chromosome. This method is based on the algorithms proposed in Section III-B, again considering the genetic model of $\mathrm{CHC}$ [29] and the steady-state approach [30] depending on the type of problem.

To do so, we must take into account the existence of binary genes (rule selection) and real values (lateral displacements) within the same chromosome. Therefore, the algorithms proposed in Section III-B are changed in order to consider a double coding scheme and to apply the appropriate genetic operators for each chromosome part. The following changes are considered in order to integrate the reduction process with the proposed tuning algorithms:

- Coding Scheme: A double coding scheme for both lateral tuning and rule selection is considered

$$
C=C_{T}+C_{S}
$$

In this case, the previous approach (part $C_{T}$ ) is combined with the rule selection by allowing an additional binary vector $C_{S}$ that determines when a rule is selected or not (alleles ' 1 ' and ' 0 ,' respectively).
Considering the $M$ rules contained in the preliminary/candidate rule set, the chromosome part $C_{S}=\left(c_{1}, \ldots, c_{M}\right)$ represents a subset of rules composing the final rule base, such that

$$
\text { IF } c_{i}=1 \text { THEN }(R i \in \mathrm{RB}) \operatorname{ELSE}(R i \notin \mathrm{RB})
$$

with $R i$ being the corresponding $i$ th rule in the candidate rule set and RB the final rule base. Fig. 8 graphically shows an example of correspondence between a chromosome and its associated $\mathrm{KB}$ considering the global approach [Fig. 8(a)] or considering the local approach [Fig. 8(b)].

- Initial Gene Pool: The initial pool is obtained with an individual having all genes with value 0.0 in the $C_{T}$ part and value 1 in the $C_{S}$ part, and the remaining individuals generated at random in $[-0.5,0.5)$ and $\{0,1\}$, respectively.

- Crossover: One of the two environment-based crossover operators presented in Section III-B for the $C_{T}$ part combined with the standard two-point crossover in the $C_{S}$ part. The two-point crossover involves exchanging the fragments of the parents contained between two points selected at random, resulting in two different offspring. In this case, four offspring are generated by combining the two from the $C_{T}$ part (environment-based crossovers) with the two from the $C_{S}$ part (two-point crossover). The two best offspring obtained in this way are considered as the two corresponding descendents.

- Mutation: When the steady-state approach is considered, a mutation operator is applied on the $C_{S}$ part of the four offspring before selecting the two descendents. This operator flips a gene value in $C_{S}$.

The application of these changes on the algorithms proposed in Section III-B gives rise to four different algorithms: $\mathrm{GL}_{d d}+$ $\mathrm{S}, \mathrm{LL}_{d d}+\mathrm{S}, \mathrm{GL}_{s s}+\mathrm{S}$ and $\mathrm{LL}_{s s}+\mathrm{S}$.

\section{EXPERIMENTAL STUDY}

To evaluate the usefulness of the proposed approaches (local and global tuning with and without rule selection for data-driven 

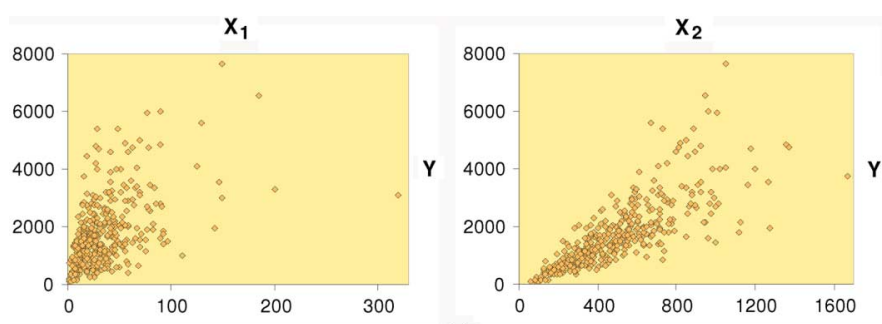

(a)
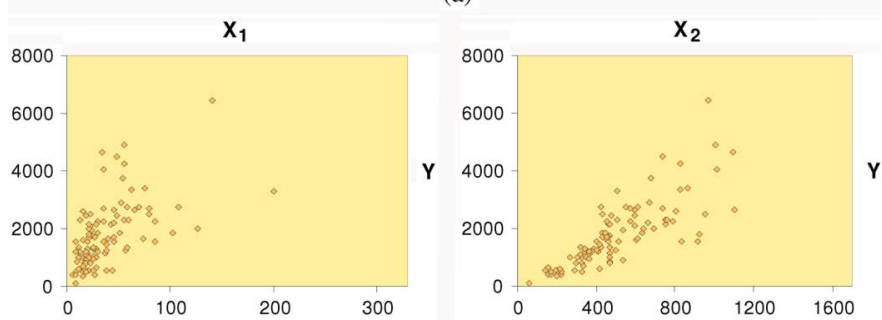

(b)

Fig. 9. (a) $\left(\mathrm{X}_{1}, \mathrm{Y}\right)$ and $\left(\mathrm{X}_{2}, \mathrm{Y}\right)$ dependency in the training data. (b) $\left(\mathrm{X}_{1}, \mathrm{Y}\right)$ and $\left(\mathrm{X}_{2}, \mathrm{Y}\right)$ dependency in the test data.

or system simulation problems) and to show their behavior regardless a single problem, several experiments have been carried out solving three real-world problems. These problems correspond to two different approaches to obtain the initial set of fuzzy rules and they have to work with a different number of variables and rules (different complexities).

- Data-Driven Problems: In this case, lateral tuning is applied to FRBSs obtained from automatic learning methods. Two real-world electrical energy distribution problems [51] are considered in order to be solved.

1) Estimating the length of low voltage lines in rural nuclei. This problem considers a small number of variables, involving a short search space (small complexity). However, the corresponding data set presents two noise points and strong nonlinearities, which makes the modeling surface very complicated.

2) Estimating the maintenance costs of medium voltage lines in a town. This problem presents four input variables and a considerable number of rules, and therefore involves a larger search space (medium complexity).

In both cases, the well-known ad hoc data-driven learning algorithm of Wang and Mendel [33] is applied to obtain an initial set of candidate linguistic rules. To do so, we will consider symmetrical fuzzy partitions of triangularshaped MFs. Once the initial RB is generated, the proposed postprocessing algorithms can be applied.

- Problem Based On System Simulations: In this case, we consider a highly complex real-world problem for the fuzzy control of an HVAC system [52]. The lateral tuning (with and without selection) will be applied to an initial fuzzy RB obtained from an expert's knowledge within the framework of a European project. The problem considers 17 variables and 172 initial fuzzy rules that make up a hierarchical fuzzy logic controller (FLC). A physical model of the controlled buildings and equipment will be considered to evaluate a chromosome.

The main achievement during the project was the application of a classic tuning method adapted to this problem
TABLE I

METHODS CONSIDERED FOR COMPARISON

\begin{tabular}{|l|c|c|}
\hline Method, Ref. & Year & Description \\
\hline WM, [33] & 1992 & Learning Method \\
S, [17] & 2000 & Rule Selection Method \\
T, [18] & 1997 & Classic Genetic Tuning \\
PAL, [49] & 2005 & Tuning of Parameters, Domains \\
& & and Local Linguistic Modifiers \\
$\mathbf{G L}_{d d}$ & - & Global Lateral Tuning \\
$\mathbf{L L}_{d d}$ & - & Local Lateral Tuning \\
\hline
\end{tabular}

[52]. The good results of this initial work motivated the study of other FRBS postprocessing methods.

In the following sections, these problems are introduced and solved to analyze the performance of the proposed techniques.

\section{A. Data-Driven Models}

Here we present the two electrical energy distribution problems [51]: the experimental setup and the results obtained by the studied algorithms.

1) Estimating the Length of Low Voltage Lines: For an electricity company, it may be of interest to measure the maintenance costs of its electricity lines. These estimations could be useful to allow them to justify their expenses. However, in some cases these costs cannot be directly calculated. The problem comes when trying to compute the maintenance costs of lowvoltage lines, and this is due to the following reasons. Although maintenance costs depend on the total length of the electrical line, the length of low-voltage lines would be very difficult and expensive to measure since they are contained in small villages and rural nuclei. The installation of these kinds of lines is often very intricate and, in some cases, one company can serve to more than 10000 rural nuclei.

Due to this reason, the length of low-voltage lines cannot be directly computed. Therefore, it must be estimated by means of indirect models. The problem involves relating the length of the low-voltage lines of a certain village with the following two variables: the radius of the village and the number of users in the village [51]. We were provided with the measured line length, the number of inhabitants, and the mean distance from the center of the town to the three furthest clients in a sample of 495 rural nuclei. Five partitions ${ }^{1}$ considering $80 \%$ (396) in training and $20 \%$ (99) in test are considered for the experiments. The existing dependency of the two input variables with the output variable in the training and test data sets in one of the five partitions is shown in Fig. 9 (notice that they present strong nonlinearities).

2) Estimating the Maintenance Costs of Medium-Voltage Lines: Estimating the maintenance costs of the medium voltage electrical network in a town [51] is a complex but interesting problem. Since a direct measure is very difficult to obtain, it is useful to consider models. These estimations allow electrical companies to justify their expenses. Moreover, the model must be able to explain how a specific value is computed for a certain town. Our objective will be to relate the maintenance costs of the medium voltage lines with the following four variables: sum of the lengths of all streets in the town, total area of the town,

\footnotetext{
${ }^{1}$ These data sets are available at http://decsai.ugr.es/ casillas/fmlib/.
} 
area that is occupied by buildings, and energy supply to the town. We will deal with estimations of minimum maintenance costs based on a model of the optimal electrical network for a town in a sample of 1059 towns. Five partitions ${ }^{1}$ considering $80 \%$ (847) in training and $20 \%$ (212) in test are considered for the experiments.

3) Experimental Setup: The methods considered for the experiments are briefly described in Table I. The use of two of them connected by "+" indicates that they are applied as a combination. For example, GL $+\mathrm{S}$ indicates global lateral tuning together with rule selection.

The WM method is considered to obtain the initial RB to be tuned. The tuning methods are applied once this initial RB has been obtained. $\mathrm{T}$ is a classic MF parameter tuning algorithm. The PAL method has been compared with tuning methods of the parameters, domain, linguistic modifiers, and with any combination of any two of them obtaining the best results [49]. For this reason, we only consider the PAL method (parameters, domain, and linguistic edges) in this paper.

The initial linguistic partitions are comprised by five linguistic terms. The fuzzy reasoning method is the minimum $t$-norm playing the role of implication and conjunctive operators and the center of gravity weighted by the matching strategy acts as defuzzification operator.

To develop the different experiments in this subsection, we consider a five-folder cross-validation model, i.e., five random partitions of data 1 each with $20 \%$, and the combination of four of them $(80 \%)$ as training and the remaining one as test. For each one of the five data partitions, the tuning methods have been run six times, showing for each problem the averaged results of a total of 30 runs. Moreover, a $t$-test (with $95 \%$ confidence) was applied in order to ascertain whether differences in the performance of the proposed approaches are significant when compared with that of the other algorithms in the respective table.

The CHC approach and MSE as fitness are considered. The following values have been considered for the parameters of each method 2 : 51 individuals, 50000 evaluations, and $\varphi=30$ bits per gene for the Gray codification $(0.2$ and 0.6 as mutation and crossover probability per chromosome and 0.35 for the factor $a$ in the max-min-arithmetical crossover for T and PAL).

4) Results: The results obtained in both problems by the analyzed methods are shown in Tables II and III, respectively, where $\# R$ stands for the number of rules, $\mathrm{MSE}_{\text {tra }}$ and $\mathrm{MSE}_{\mathrm{tst}}$, respectively, for the averaged error obtained over the training and test data, and $\sigma$ for the standard deviation. $t$-test represents the following information:

$$
\begin{aligned}
& \text { * represents the best averaged result. } \\
& +\quad \text { means that the best result has better performance } \\
& \text { than that of the corresponding row. } \\
& =\quad \begin{array}{l}
\text { denotes that the results are statistically equal } \\
\text { according to the } t \text {-test. }
\end{array}
\end{aligned}
$$

\footnotetext{
${ }^{2}$ With these values we have tried to ease the comparisons by selecting standard common parameters that work well in most cases, instead of searching for very specific values for each method. Moreover, we have set a large number of evaluations in order to allow the compared algorithms to achieve an appropriate

\begin{tabular}{|c|c|c|c|c|c|c|c|}
\hline Method & \#R & $\mathbf{M S E}_{t r a}$ & $\sigma_{t r a}$ & t-test & $\mathbf{M S E}_{t s t}$ & $\sigma_{t s t}$ & t-test \\
\hline \multicolumn{8}{|c|}{ Approaches without tuning } \\
\hline WM & 12.4 & 234712 & 32073 & + & 242147 & 24473 & + \\
\hline S & 10.0 & 226135 & 19875 & + & 241883 & 19410 & + \\
\hline \multicolumn{8}{|c|}{ Approaches with global semantics } \\
\hline $\mathrm{T}$ & 12.4 & 158662 & 6495 & + & 221613 & 29986 & + \\
\hline $\mathrm{T}+\mathrm{S}$ & 8.9 & 156313 & 2967 & + & 193477 & 49912 & $=$ \\
\hline $\mathrm{GL}_{d d}$ & 12.4 & 166674 & 11480 & + & 189216 & 14743 & $=$ \\
\hline $\mathrm{GL}_{d d}+\mathrm{S}$ & 9.0 & 160081 & 7316 & + & 189844 & 22448 & $=$ \\
\hline \multicolumn{8}{|c|}{ Approaches with local semantics } \\
\hline PAL & 12.4 & 141638 & 4340 & + & 189279 & 19523 & $=$ \\
\hline $\mathrm{PAL}+\mathrm{S}$ & 10.6 & 145712 & 5444 & + & 191922 & 16987 & $=$ \\
\hline $\mathrm{LL}_{d d}$ & 12.4 & 139189 & 3155 & $\star$ & 191604 & 18243 & $=$ \\
\hline $\mathrm{LL}_{d d}+\mathrm{S}$ & 10.5 & 141446 & 3444 & $=$ & 186746 & 15762 & $\star$ \\
\hline
\end{tabular}
convergence. No significant changes were achieved by increasing that number of evaluations.
}

TABLE II

\begin{tabular}{|c|c|c|c|c|c|c|c|}
\hline Method & $\# \mathbf{R}$ & $\mathbf{M S E}_{t r a}$ & $\sigma_{t r a}$ & t-test & $\mathbf{M S E}_{t s t}$ & $\sigma_{t s t}$ & t-test \\
\hline \multicolumn{8}{|c|}{ Approaches without tuning } \\
\hline WM & 65 & 57605 & 2841 & + & 57934 & 4733 & + \\
\hline $\mathrm{S}$ & 40.8 & 41086 & 1322 & + & 59942 & 4931 & + \\
\hline \multicolumn{8}{|c|}{ Approaches with global semantics } \\
\hline $\mathrm{T}$ & 65 & 18602 & 1211 & + & 22666 & 3386 & + \\
\hline $\mathrm{T}+\mathrm{S}$ & 41.9 & 14987 & 391 & + & 18973 & 3772 & + \\
\hline $\mathrm{GL}_{d d}$ & 65 & 23064 & 1479 & + & 25654 & 2611 & + \\
\hline $\mathrm{GL}_{d d}+\mathrm{S}$ & 49.1 & 18801 & 2669 & + & 22586 & 3550 & + \\
\hline \multicolumn{8}{|c|}{ Approaches with local semantics } \\
\hline PAL & 65 & 10545 & 279 & + & 13973 & 1688 & + \\
\hline $\mathrm{PAL}+\mathrm{S}$ & 57.4 & 12851 & 362 & + & 16854 & 1463 & + \\
\hline $\mathrm{LL}_{d d}$ & 65 & 3664 & 390 & $\star$ & 5858 & 1798 & 夫 \\
\hline $\mathrm{LL}_{d d}+\mathrm{S}$ & 58.0 & 3821 & 385 & $=$ & 6339 & 2164 & $=$ \\
\hline
\end{tabular}

ResUlts OBtained IN THE LiNE LENGTH Estimation PROBLEM

TABLE III

Results Obtained in the Maintenance Costs Estimation Problem

Analyzing the results presented in Tables II and III, we can highlight the following conclusions.

- Considering these methods, the RB is obtained by means of a generation method to learn few rules (12.4 from 25, 65 from the 625 possible rules). It allows us to obtain a compact and accurate tuned model.

- In the first problem, low-voltage line length estimation, most of the methods show a similar error in the test partitions. This performance is due to the small number of variables considered in this problem, which means that the search space is not too complex and therefore, there are no significant differences in the tuning methods. However, in the training partitions, the local lateral tuning obtains better results than the remaining approaches, but some of these differences are not so important. In other words, in a problem with small complexity in the search space, the lateral tuning still does not have the ability to take an advantage over the remaining approaches.

- In the second problem, which handles more variables and therefore involves a larger search space, the local lateral tuning method shows an important reduction of the MSE. This is due to the use of only one parameter per label, reducing the search space with respect to a classic tuning which usually considers three or four parameters (in the 


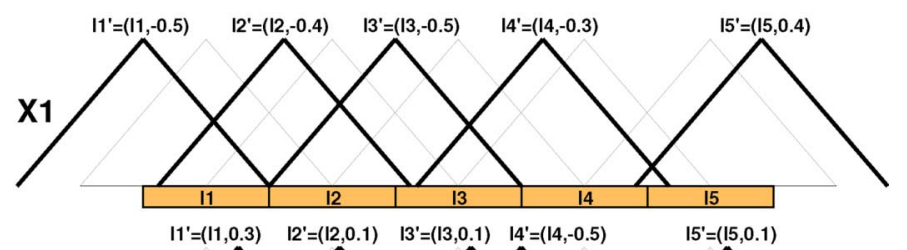

X2
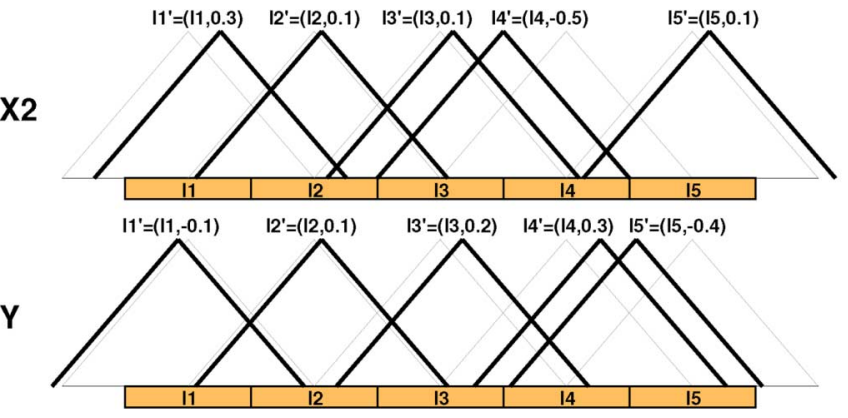

Fig. 10. Initial and tuned $\mathrm{DB}$ of a model obtained with $\mathrm{GL}_{d d}$ in the low-voltage line length estimation problem.

case of triangular or trapezoidal MFs). In this way, the local lateral tuning presents a good relationship between the search space complexity and the results obtained, which maintain a high tradeoff between accuracy and local interpretability in the method. Furthermore, since the lateral displacements are related to the original global labels, a global interpretation could be made in these terms.

- The global lateral tuning method shows worse results than some of the classic approaches. However, in the following problem (HVAC system control) with increased complexity and a larger number of variables, this method achieves very good results, even better than those obtained by the local approach and with a better global interpretability. This could again be due to the search space reduction, since this approach handles an even smaller number of parameters than the local one.

- With regard to the combination of tuning and the selection methods, in both problems, GL $+\mathrm{S}$ and LL $+\mathrm{S}$, respectively, show more or less the same accuracy as GL and LL, considering a reduced number of rules and therefore reaching a good tradeoff between both techniques. Notice that the combination of several techniques (especially when they act on different parts of the FRBS) increases the search space complexity. However, in the case of the global lateral tuning, the accuracy even improves when it is combined with the selection approach.

Figs. 10 and 11, respectively, present the evolved fuzzy linguistic partitions and the decision table obtained by $\mathrm{GL}_{d d}$ from one of the 30 runs performed in the first problem. Figs. 12 and 13 , respectively, present the evolved fuzzy linguistic partitions and the RB obtained by $\mathrm{GL}_{d d}$ from one of the 30 runs performed in the second problem. These figures show that small variations in the MFs lead to important improvements in the performance of the obtained FRBSs.

Fig. 14 graphically shows one of the $30 \mathrm{RBs}$ obtained with $\mathrm{LL}_{d d}$ in the second problem, where we can see how local tuning evolves each label of the different rules in a different way. The difficult tradeoff between accuracy and complexity can be ob-

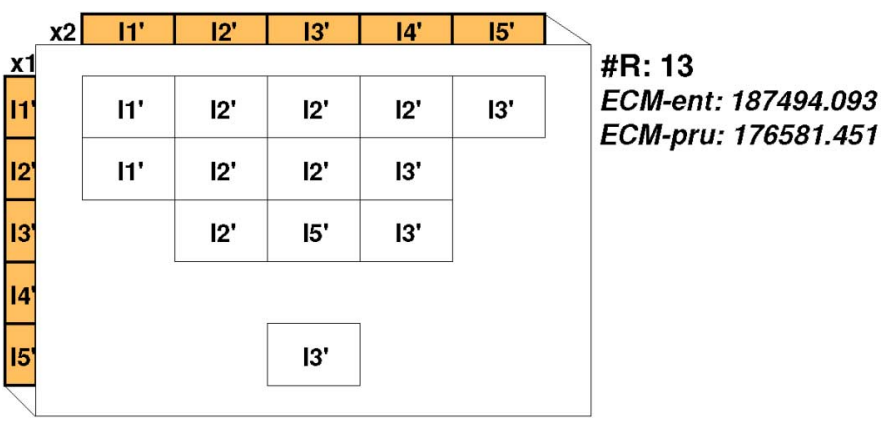

Fig. 11. Decision table of a model obtained with $\mathrm{GL}_{d d}$ in the low-voltage line length estimation problem.

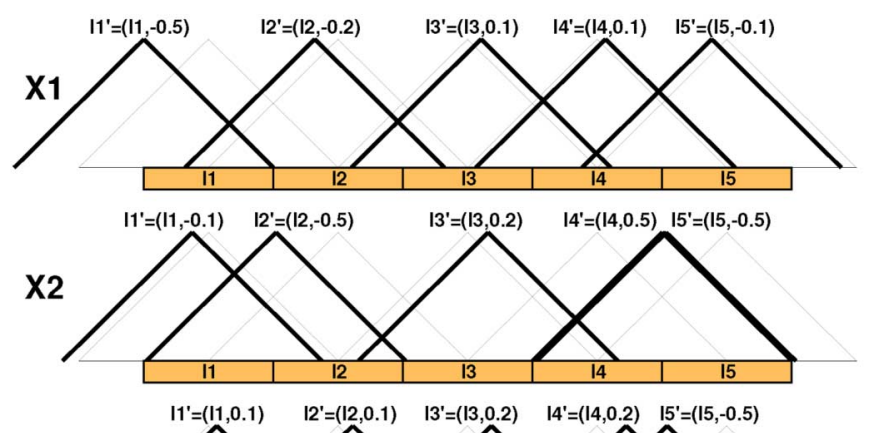

$\mathbf{X} 3$
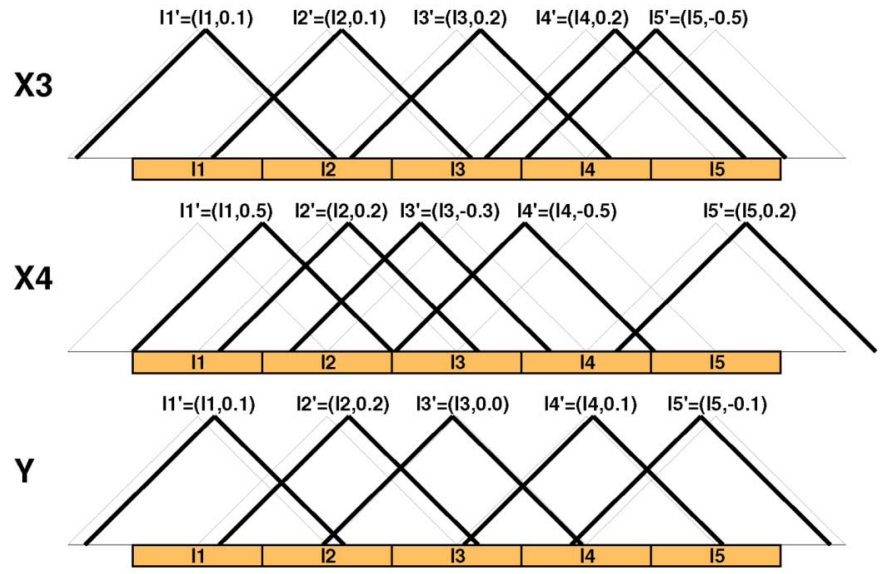

Fig. 12. Initial and tuned $\mathrm{DB}$ of a model obtained with $\mathrm{GL}_{d d}$ in the maintenance costs estimation problem.

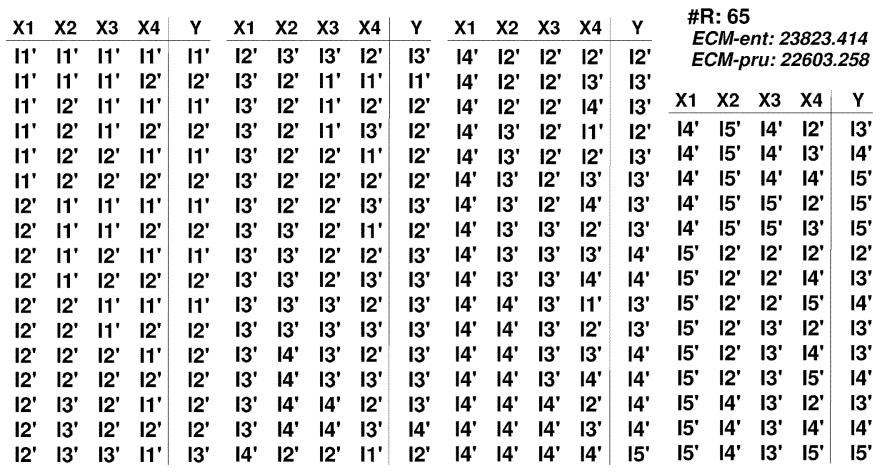

Fig. 13. RB and lateral displacements of a model obtained with $\mathrm{GL}_{d d}$ in the maintenance costs estimation problem.

served taking into account both RBs (Figs. 13 and 14). The accuracy can be improved but always at the expense of some interpretability. 


\begin{abstract}
\#R: 65
\begin{tabular}{lllll|l}
$\mathbf{X} 1$ & $\mathbf{X} 2$ & $\mathbf{X} 3$ & $\mathbf{X} 4$ & $\mathrm{Y}$ \\
\hline
\end{tabular}

\begin{tabular}{lllll}
\hline $1+0.24$ & $\mathbf{I 1}+0.36 \quad \mathbf{I 1}-0.44 \quad \mathbf{I 1 + 0 . 1 6}$ & $\mathbf{I 1}-0.34$
\end{tabular}

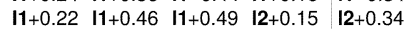

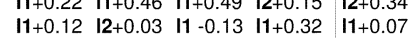
$\begin{array}{llllll}\mathbf{I}++0.12 & \mathbf{I}+0.03 & \mathbf{I} & -0.13 & \mathbf{I} \mathbf{1}+0.32 & \mathbf{I} \mathbf{1}+0.07 \\ \mathbf{I}+0.44 & \mathbf{I}+0.34 & \mathbf{1}-0.44 & \mathbf{I}+0.46 & \mathbf{I} \mathbf{2}-0.48\end{array}$

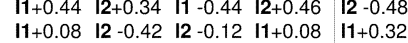
$\begin{array}{lllllll}\mathbf{1 1}+0.28 & \mathbf{1 2} & -0.08 & \mathbf{1 2} & -0.32 & \mathbf{1 2}+0.20 & \mathbf{1 2}+0.40\end{array}$ $\begin{array}{lllllll}\mathbf{I 2}-0.07 & \mathbf{I 1}+0.04 & \mathbf{1 1}-0.38 & \mathbf{I}+\mathbf{1}+0.34 & \mathbf{I 1}+0.10\end{array}$

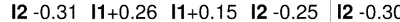
$\begin{array}{llllll}\mathbf{1 2}+0.02 \quad \mathbf{I} & -0.46 \quad \mathbf{2}+0.46 \quad \mathbf{I} & -0.33 \quad \mathbf{I}+0.29\end{array}$ $\begin{array}{ll}12 & -0.49 \quad \mathbf{I 1}-0.09 \quad 12+0.16 \quad 12+0.00 \quad 12-0.03\end{array}$ $12-0.4911-0.0912+0.16 \quad 12+0.00 \quad 12-0.03$ $\begin{array}{llllll}\mathbf{I}+0.15 & \mathbf{I}+0.02 & \mathbf{I 1}+0.33 \quad \mathbf{I} & -0.25 & \mathbf{I 1}+0.12\end{array}$ $\begin{array}{llllllll}\mathbf{1 2} & -0.31 & \mathbf{1 2}+0.43 & \mathbf{1 1} & -0.04 & \mathbf{1 2}+0.21 & \mathbf{1 2} & -0.35\end{array}$ $\begin{array}{llllll}\mathbf{1 2}+0.27 & \mathbf{1 2}+0.25 & \mathbf{1 2}+0.33 & \mathbf{1} 1+0.39 & \mathbf{I} 2+0.16\end{array}$ $\begin{array}{lllllll}\mathbf{1 2}-0.10 & \mathbf{1 2} & -0.36 & \mathbf{I 2}+0.04 & \mathbf{1 2}+0.13 & \mathbf{I 2}+0.47\end{array}$

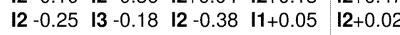
$\begin{array}{llllllll}\mathbf{1 2}+0.37 & \mathbf{1 3} & -0.33 & \mathbf{1 2} & -0.19 & \mathbf{1 2}+0.26 & \mathbf{1 2} & -0.21\end{array}$

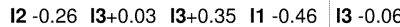
$\begin{array}{lllllll}12 & +0.47 \quad \mathbf{3} & -0.22 \quad \mathbf{3} & -0.20 & \mathbf{1} 2 & -0.37 & \mathbf{1 3}+0.16\end{array}$ $13-0.02 \quad 12-0.43 \quad 11-0.2011-0.42 \quad 11-0.05$

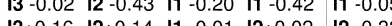
$\begin{array}{lllllll}\mathbf{1 3}+0.16 & \mathbf{I 2}+0.14 & \mathbf{1 1} & -0.01 & \mathbf{I 2}+0.02 & \mathbf{1 2} & -0.33\end{array}$

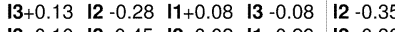
$\begin{array}{lllllll}\mathbf{1 3}+0.10 & \mathbf{1 2}+0.45 & \mathbf{1 2}+0.02 & \mathbf{1 1} & -0.29 & \mathbf{I} 2 & -0.30\end{array}$ $\begin{array}{llllll}\mathbf{1 3}+0.03 & \mathbf{1 2}+0.35 & \mathbf{1 2}-0.34 & \mathbf{1 2}+0.16 & \mathbf{1 2}+0.19\end{array}$ $\begin{array}{llllll}\mathbf{1 3}+0.15 & \mathbf{1 2}-0.33 & \mathbf{1 2}+0.04 & \mathbf{1 3}+0.04 & \mathbf{1 3}+0.02\end{array}$ $\begin{array}{lllllll}\mathbf{1 3}+0.11 & \mathbf{1 3}+0.44 & \mathbf{1 2}+0.17 & \mathbf{1 1}+0.39 & \mathbf{1 2}+0.18\end{array}$ $\begin{array}{lllllll}13 & 0.00 & \mathbf{1 3}+0.06 & \mathbf{1 2}+0.37 & \mathbf{1 2}+0.26 & \mathbf{1 3} & -0.50\end{array}$ $\begin{array}{llllll}13 & -0.50 \quad 13 & -0.43 & \mathbf{1 2}+0.37 \quad & \mathbf{1 3}+0.10 \quad & \mathbf{3}+0.44\end{array}$ $\begin{array}{lll}13-0.50 & 13-0.43 \quad 12+0.37 \quad 13+0.10 \quad 13+0.44\end{array}$ $\begin{array}{llllll}13 & -0.29 & \mathbf{3} 3+0.18 & \mathbf{1 3}+0.40 & \mathbf{1} \mathbf{2}+0.06 & \mathbf{3}+0.15\end{array}$

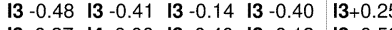
\begin{tabular}{lllll|l}
$\mathbf{I 3}+0.37$ & $\mathbf{I 4}+0.06$ & $\mathbf{I 3}+0.40$ & $\mathbf{I} 2-0.13$ & $\mathbf{I 3}+0.50$ \\
$\mathbf{1 3}+0.48$ & $\mathbf{1 4}+0.34$ & $\mathbf{1 3}-0.31$ & $\mathbf{I 3}+0.45$ & $\mathbf{I 3}-0.15$
\end{tabular}

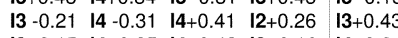
$\begin{array}{llllllllll}13 & -0.15 & \mathbf{1 4} & -0.25 & \mathbf{I 4} & -0.43 & \mathbf{I 3} & -0.16 & \mathbf{1 4} & -0.04\end{array}$

ECM-ent: 3085.859

ECM-ent: 3085.859
ECM-pru: 4124.033
\end{abstract}

Fig. 14. RB and lateral displacements of a model obtained with $\mathrm{LL}_{d d}$ in the maintenance costs estimation problem.

To ease graphic representation, in all these figures, the MFs are labeled from ' 11 ' to ' $1 L^{i}$. Nevertheless, such MFs are associated to a linguistic meaning determined by an expert. In this way, if the ' 11 ' label of the ' $\mathrm{X} 1$ ' variable represents 'LOW,' then ' $l 1+0.11$ ' could be interpreted as 'a little smaller than LOW' (based on the expert opinion) or, as in the case of the classic tuning approach, maintaining the original meaning of such label. This is the case of Figs. 10 and 12, where practically all the new labels could maintain their initial meanings.

A graphical representation of the proposed algorithms convergence on both problems is shown in Fig. 15 with respect to the training data. As can be seen, in general this figure shows similar performances of the global approaches and similar performances of the local ones in both problems. Moreover, we can highlight the fast improvement in the problem with a smaller search space and the more gradual improvements in a problem with a larger search space, in which finding a good solution is much more difficult. This reflects the ability of the algorithms to find a good search zone in which to find a more global solution by refining the parameters more and more, thereby avoiding getting stuck in a local optimum.

A study on the influence of the parameter $\varphi$ (number of bits per gene for the Gray codification) is shown in Table IV. The results in the table are again the averaged MSE of 30 runs in training and test with different $\varphi$ values for each algorithm. In this case, on one hand, the t-test was applied to the different results obtained by the $G L_{d d}$ algorithm, and on the other hand to the different results obtained by the $L L_{d d}$ algorithm in order to compare the results obtained with different values of the parameter $\varphi$. As can be seen, considering different values of $\varphi$ does not significantly affect the results of both methods, which shows the robustness of the algorithms proposed for the lateral tuning of MFs. We can only highlight that the use of a very low $\varphi$ value provoked a worse result in the case of the problem with a higher search space. Therefore, we can only recommend a not too small value for this parameter. A study on the parameter related to the GA (population size) has been not performed since there are different studies on these parameters and we consider general values that have demonstrated a good performance in different problems (these values are the same in the different algorithms considered for comparisons).

\section{B. Expert Knowledge Based Models}

In this section, we apply lateral tuning (with and without rule selection) to develop accurate FLCs dedicated to the intelligent control of HVAC systems concerning energy performance and indoor comfort requirements. First, the HVAC system control problem is introduced, and then the experimental study is presented. In the Appendix, the basics of the HVAC systems control problem are presented together with the real test site, the control objectives, and the fitness function of our specific application.

1) Tuning of FLCs for HVAC Systems: In European Union countries, primary energy consumption in buildings represents about $40 \%$ of total energy consumption, and more than half of this energy is used for indoor climate conditions. From a technological point of view, it is estimated that the consideration of specific technologies like building energy management systems (BEMSs) can save up to $20 \%$ of the energy consumption of the building sector, i.e., $8 \%$ of the overall European Community consumption [53]. With this aim, BEMSs are generally applied only to the control of active systems, i.e., HVAC systems.

HVAC systems are equipment usually implemented for maintaining satisfactory conditions of comfort in buildings. Energy consumption as well as indoor comfort aspects of ventilated and air conditioned buildings are highly dependent on the design, performance, and control of their HVAC systems and equipment. Therefore, the use of appropriate automatic control strategies, such as fuzzy logic controllers (FLCs) [4], [5], [13], for HVAC systems control could result in important energy savings when compared to manual control [52], [54].

In current systems [54]-[62], various criteria are considered under separate cover, thermal regulation, maintaining a temperature set point or range, energy consumption, predicted mean vote (PMV), system stability, or indoor air quality. Moreover, control systems in buildings are often designed using rules of thumb not always compatible with the controlled equipment requirements and energy performance. Therefore, the different criteria involved should be optimized for a good performance of the HVAC system. Usually, the main objective is to reduce the energy consumption while maintaining a desired comfort level. In our case, five criteria will be optimized, and 17 variables are considered by the FLC.

The initial FLC to be optimized is obtained from human experience. This approach is tested considering the calibrated and validated models of a real test building. Both the FLC and the simulation model were developed within the framework of the JOULE-THERMIE programme under the GENESYS project. ${ }^{3}$

${ }^{3}$ Fuzzy controllers and smart tuning techniques for energy efficiency and overall performance of HVAC systems in buildings, European Commission, Directorate-General XII for Energy (Contract JOE-CT98-0090). 

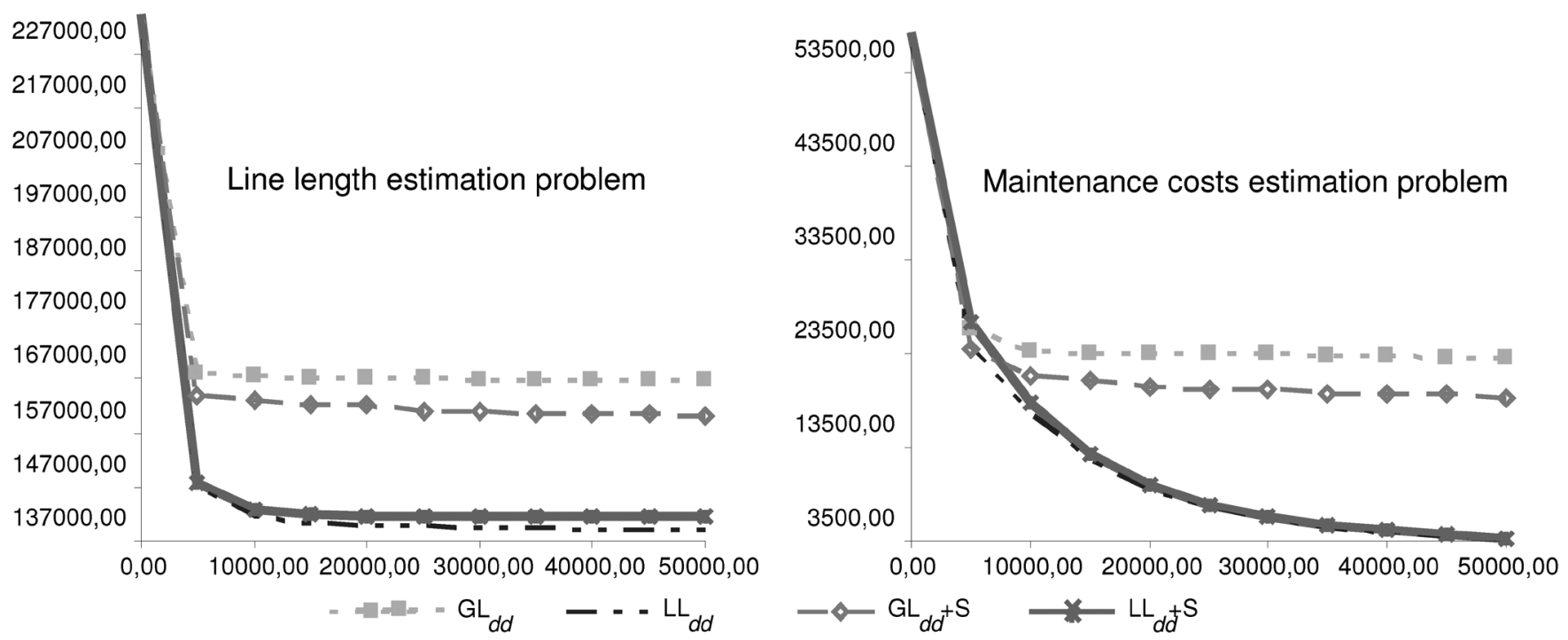

Fig. 15. Convergence of the proposed methods considering the averaged results each 5000 evaluations.

TABLE IV

Study on the Parameter $\varphi$ (Number of Bits Per Gene for the Gray Codification)

\begin{tabular}{|c|c|c|c|c|c|c|c|c|c|c|c|c|c|}
\hline & \multicolumn{7}{|c|}{ The line length estimation problem } & \multicolumn{6}{|c|}{ The maintenance costs estimation problem } \\
\hline Method & $\varphi$ & $\mathbf{M S E}_{t r a}$ & $\sigma_{t r a}$ & t-test & $\mathbf{M S E}_{t s t}$ & $\sigma_{t s t}$ & t-test & $\mathbf{M S E}_{t r a}$ & $\sigma_{t r a}$ & t-test & $\mathbf{M S E}_{t s t}$ & $\sigma_{t s t}$ & t-test \\
\hline $\mathrm{GL}_{d d}$ & 10 & 167116 & 11271 & $=$ & 190161 & 15205 & $=$ & 23458 & 2533 & $=$ & 26084 & 3115 & $=$ \\
\hline $\mathrm{GL}_{d d}$ & 20 & 166905 & 11339 & $=$ & 187734 & 16835 & $*$ & 23879 & 3033 & $=$ & 26630 & 4005 & $=$ \\
\hline $\mathrm{GL}_{d d}$ & 30 & 166674 & 11480 & $=$ & 189216 & 14743 & $=$ & 23064 & 1479 & $*$ & 25654 & 2611 & $*$ \\
\hline $\mathrm{GL}_{d d}$ & 40 & 166382 & 11534 & $*$ & 189816 & 15342 & $=$ & 23851 & 2302 & $=$ & 26871 & 3273 & $=$ \\
\hline $\mathrm{GL}_{d d}$ & 50 & 166669 & 11450 & $=$ & 188967 & 15707 & $=$ & 23658 & 1914 & $=$ & 26535 & 4107 & $=$ \\
\hline $\mathrm{LL}_{d d}$ & 10 & 139261 & 2848 & $=$ & 190072 & 15755 & $*$ & 4043 & 467 & + & 6713 & 1658 & $=$ \\
\hline $\mathrm{LL}_{d d}$ & 20 & 139253 & 4821 & $=$ & 193059 & 19280 & $=$ & 3690 & 310 & $=$ & 5866 & 1523 & $=$ \\
\hline $\mathrm{LL}_{d d}$ & 30 & 139189 & 3155 & $*$ & 191604 & 18243 & $=$ & 3664 & 390 & $=$ & 5858 & 1798 & $=$ \\
\hline $\mathrm{LL}_{d d}$ & 40 & 140436 & 4205 & $=$ & 194427 & 23765 & $=$ & 3522 & 425 & $*$ & 5407 & 1049 & $*$ \\
\hline $\mathrm{LL}_{d d}$ & 50 & 139555 & 2685 & $=$ & 191749 & 18423 & $=$ & 3591 & 360 & $=$ & 6235 & 2663 & $=$ \\
\hline
\end{tabular}

From now on, this test building will be called the GENESYS test site.

In the Appendix, the main aspects of the HVAC systems and their control with FLCs are described. Furthermore, the GENESYS test site, the control objectives, and the initial DB and RB are presented. In this section, we apply the lateral tuning approaches to improve the performance of this initial FLC by considering the simulation model of the GENESYS test site.

2) Experimental Setup: To evaluate the usefulness of the proposed technique, several experiments have been carried out considering the GENESYS test site. The methods considered for the experiments are briefly described in Table V. The use of two of them connected by ' + ' indicates that they are applied as a combination. For example, $\mathrm{GL}+\mathrm{S}$ indicates global lateral tuning together with rule selection. Methods $\mathrm{S}, \mathrm{C}$, and $\mathrm{C}+\mathrm{S}$ were adapted from [17], [12], and [48], respectively, in order to be applied to this problem and to follow the steady-state approach presented in [52]. As can be seen, there are no local semantics-based approaches other than those proposed in this paper since, due to the high-dimensional search space, these kinds of methods never have been applied in this particular problem.
The FLCs obtained by the studied methods will be compared with the performance of the initial FLC and with the performance of a classic on-off controller. The goals and improvements will be computed with respect to this classic controller as in the GENESYS project. The experts' aim was to try to obtain a $10 \%$ energy saving $\left(\mathrm{O}_{4}\right)$ together with a global improvement of the system performance compared to on-off control. Comfort parameters could be slightly increased if necessary (no more than 1.0 for criteria $O_{1}$ and $O_{2}$ ).

Table VI presents the results obtained with the on-off and the initial FLC controllers together with the parameters considered to compute the fitness function in the GA $\left(F^{\prime}\right)$, fuzzy goals, and penalization rates (for more information on these parameters, see Appendix B1). Notice that the goals imposed to the algorithm are higher than those initially required by the experts since we are trying to obtain even better results. No improvement percentages have been considered in the table for $O_{1} \cdots O_{3}$, since these objectives always met the experts' requirements and the on-off controller presents zero values for these objectives.

Finally, the values of the parameters used in all of these experiments are presented as follows: 2000 evaluations, 31 individuals, and 0.3 for the factor $\alpha$ in the BLX crossover operator 
TABLE V

METHODS CONSIDERED FOR COMPARISON

\begin{tabular}{|l|c|c|}
\hline Method, Ref. & Year & Description \\
\hline S, [63] & 2005 & Rule Selection $\left(C_{S}\right.$ part of GL+S) \\
C, $[52]$ & 2003 & Genetic Classic Tuning \\
GL $_{s s},-$ & - & Global Lateral-tuning \\
$\mathbf{L L}_{s s},-$ & - & Local Lateral-tuning \\
\hline
\end{tabular}

TABLE VI

INITIAL Results AND Fitness FunCtion $\left(F^{\prime}\right)$ PARAMETERS

\begin{tabular}{ccccccccc}
\hline & & \multicolumn{2}{c}{$\mathrm{PMV}$} & $\mathrm{CO}_{2}$ & \multicolumn{2}{c}{ Energy } & \multicolumn{2}{c}{ Stability } \\
MODEL & $\# \mathrm{R}$ & $O_{1}$ & $\mathrm{O}_{2}$ & $O_{3}$ & $O_{4}$ & $\%$ & $O_{5}$ & $\%$ \\
\hline ON-OFF & - & 0.0 & 0 & 0 & 3206400 & - & 1136 & - \\
INIT. FLC & 172 & 0.0 & 0 & 0 & 2901686 & 9.50 & 1505 & -32.48 \\
\hline Goals $\left(g_{i}\right)$ & - & 1.0 & 1 & 7 & 2000000 & - & 1000 & - \\
Rates $\left(p_{i}\right)$ & - & 1 & 1 & 1 & 0.9 & - & 0.97 & - \\
\hline
\end{tabular}

( 0.2 as mutation probability per chromosome for $\mathrm{C}+\mathrm{S}, \mathrm{GL}+\mathrm{S}$, and $\mathrm{LL}+\mathrm{S}$ ). In order to evaluate the good convergence of the GA, three different runs have been performed considering three different seeds for the random number generator.

The proposed optimization strategy was assessed with simulations of ten days with the corresponding climatic conditions (with a computation time of approximately 2 min per simulation). Therefore, the estimated run time is approximately three days for each run. This is the reason why in this case the steadystate approach is considered.

3) Results: The models presented in Table VII, where "\%" stands for the improvement rate with respect to the on-off controller for each criterion and "\#R" the number of fuzzy rules, correspond to the best individuals from the population at evaluation 2000 in the three runs performed. The averaged results for each criterion are shown in Table VIII.

Analyzing the results obtained, we can highlight the following conclusions.

- The global lateral tuning method improves the performance of the obtained linguistic models with respect to energy consumption, especially when it is combined with the rule selection, which also improves the system stability.

- Local lateral tuning together with rule selection obtains a linguistic model with the highest level of system stability, maintaining a high level of energy saving.

- The combination of lateral tuning (global and local approach) with the rule selection allows us to remove many redundant, erroneous, and conflictive rules, greatly improving the system performance with respect to the individual use of the tuning algorithms.

- The classic tuning of MF is a generalization of lateral tuning. Therefore, why does lateral tuning present much better results than classic tuning?

The tuning of an FRBS for fuzzy control of HVAC systems is a very complex problem with 17 variables, and the use of only one parameter per MF eases the derivation of an optimal model with respect to the classic tuning with three parameters per MF. This effect is even more notable when
TABLE VII

RESUlts OBTAINED BY THE METHODS CONSIDERED

\begin{tabular}{|c|c|c|c|c|c|c|c|c|}
\hline \multirow{2}{*}{ MODEL } & \multirow[b]{2}{*}{ \#R } & \multicolumn{2}{|c|}{ PMV } & \multirow{2}{*}{$\begin{array}{r}\mathrm{CO}_{2} \\
\mathrm{O}_{3} \\
\end{array}$} & \multicolumn{2}{|c|}{ Energy } & \multirow{2}{*}{\multicolumn{2}{|c|}{$\begin{array}{l}\text { Stability } \\
O_{5} \% \%\end{array}$}} \\
\hline & & $O_{1}$ & $\mathrm{O}_{2}$ & & $\mathrm{O}_{4}$ & $\%$ & & \\
\hline \multicolumn{9}{|c|}{ Rule Selection } \\
\hline S1 & 147 & 0.2 & 0 & 0 & 2867692 & 10.56 & 991 & 12.76 \\
\hline S2 & 162 & 0.0 & 0 & 0 & 2889889 & 9.87 & 1441 & -26.85 \\
\hline S3 & 172 & 0.0 & 0 & 0 & 2901686 & 9.50 & 1505 & -32.48 \\
\hline \multicolumn{9}{|c|}{ Classic Tuning } \\
\hline $\mathrm{C} 1$ & 172 & 0.0 & 0 & 0 & 2575949 & 19.66 & 1115 & 1.85 \\
\hline $\mathrm{C} 2$ & 172 & 0.0 & 0 & 0 & 2587326 & 19.31 & 1077 & 5.19 \\
\hline $\mathrm{C} 3$ & 172 & 0.0 & 0 & 0 & 2596875 & 19.01 & 1051 & 7.48 \\
\hline \multicolumn{9}{|c|}{ Selection with Classic Tuning } \\
\hline $\mathrm{C}+\mathrm{S} 1$ & 94 & 0.0 & 0 & 0 & 2540065 & 20.78 & 1294 & -13.91 \\
\hline $\mathrm{C}+\mathrm{S} 2$ & 109 & 0.1 & 0 & 0 & 2492462 & 22.27 & 989 & 12.94 \\
\hline $\mathrm{C}+\mathrm{S} 3$ & 100 & 0.1 & 0 & 0 & 2578019 & 19.60 & 887 & 21.92 \\
\hline \multicolumn{9}{|c|}{ Global Lateral Tuning } \\
\hline $\mathrm{GL}_{s s} 1$ & 172 & 0.7 & 0 & 0 & 2378784 & 25.81 & 1069 & 5.90 \\
\hline $\mathrm{GL}_{s s} 2$ & 172 & 1.0 & 0 & 0 & 2327806 & 27.40 & 1066 & 6.16 \\
\hline $\mathrm{GL}_{s s} 3$ & 172 & 0.9 & 0 & 0 & 2268689 & 29.25 & 1080 & 4.93 \\
\hline \multicolumn{9}{|c|}{ Local Lateral Tuning } \\
\hline $\mathrm{LL}_{s s} 1$ & 172 & 0.9 & 0 & 0 & 2386033 & 25.59 & 896 & 21.13 \\
\hline $\mathrm{LL}_{s s} 2$ & 172 & 0.8 & 0 & 0 & 2343409 & 26.92 & 943 & 16.99 \\
\hline $\mathrm{LL}_{s s} 3$ & 172 & 0.3 & 0 & 0 & 2377596 & 25.85 & 938 & 17.43 \\
\hline \multicolumn{9}{|c|}{ Selection with Global Lateral Tuning } \\
\hline $\mathrm{GL}_{s s}+\mathrm{S} 1$ & 105 & 1.0 & 0 & 0 & 2218598 & 30.81 & 710 & 37.50 \\
\hline $\mathrm{GL}_{s s}+\mathrm{S} 2$ & 115 & 0.4 & 0 & 0 & 2358405 & 26.45 & 818 & 27.99 \\
\hline $\mathrm{GL}_{s s}+\mathrm{S} 3$ & 118 & 0.8 & 0 & 0 & 2286976 & 28.68 & 872 & 23.24 \\
\hline \multicolumn{9}{|c|}{ Selection with Local Lateral Tuning } \\
\hline$+\mathrm{S} 1$ & 133 & 0.5 & 0 & 0 & 2311986 & 27.90 & 788 & 30.63 \\
\hline $\mathrm{LL}_{s s}+\mathrm{S} 2$ & 104 & 0.6 & 0 & 0 & 2388470 & 25.51 & 595 & 47.62 \\
\hline $\mathrm{LL}_{s s}+\mathrm{S} 3$ & 93 & 0.5 & 0 & 0 & 2277807 & 28.96 & 1028 & 9.51 \\
\hline
\end{tabular}

TABLE VIII

Averaged Results of THE Methods Considered

\begin{tabular}{|c|c|c|c|c|c|c|c|c|}
\hline MODEL & $\# \mathrm{R}$ & \multicolumn{2}{|c|}{ PMV } & $\begin{array}{r}\mathrm{CO}_{2} \\
\mathrm{O}_{3} \\
\end{array}$ & \multicolumn{2}{|c|}{ Energy } & \multicolumn{2}{|c|}{$\begin{array}{l}\text { Stability } \\
O_{5} \%\end{array}$} \\
\hline $\mathrm{ON}-$ & - & 0.0 & 0 & 0 & 3206400 & - & 1136 & - \\
\hline \multicolumn{9}{|c|}{ Approaches without tuning } \\
\hline NIT. FLC & 172 & 0.0 & 0 & 0 & 2901686 & 9.50 & 1505 & -32.48 \\
\hline & 160 & 0.1 & 0 & 0 & 2886422 & 9.9 & 312 & -15.52 \\
\hline \multicolumn{9}{|c|}{ Approaches with global semantics } \\
\hline $\bar{C}$ & 172 & 0.0 & 0 & 0 & 2586717 & 19.33 & 1081 & 4.84 \\
\hline$\overline{C+S}$ & 101 & 0.1 & 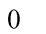 & 0 & 2536 & 20 & 1057 & 6.98 \\
\hline$\overline{G L_{s s}}$ & 17 & 0. & 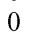 & 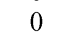 & & & 1072 & 5.66 \\
\hline$L_{s s}+S$ & 113 & 0.7 & 0 & 0 & 2287993 & 28.64 & 800 & 29.58 \\
\hline \multicolumn{9}{|c|}{ Approaches with local semantics } \\
\hline$\overline{L L_{s s}}$ & 172 & 0.7 & 0 & 0 & 2369013 & 26.1 & 926 & 18.52 \\
\hline$L L_{s s}+S$ & 110 & 0.5 & 0 & 0 & 2326088 & 27.46 & 804 & 29.26 \\
\hline
\end{tabular}

rule selection is also considered, since it involves a larger search space.

Fig. 16 represents the initial and the final DBs obtained by the first run of $\mathrm{GL}_{\mathrm{ss}}+\mathrm{S}$. Again, small variations in the MF parameters cause large improvements in the linguistic model performance. Fig. 17 represents the corresponding decision tables of the model obtained from $\mathrm{GL}_{s s}+\mathrm{S}$ considering the first seed (for an explanation of these kinds of figures, see Appendix B2). In this case, a large number of rules have been removed from the initial FLC, obtaining much simpler models (more or less 59 rules were eliminated in each run). This fact improves the system readability and allows us to obtain simple and accurate FLCs. 


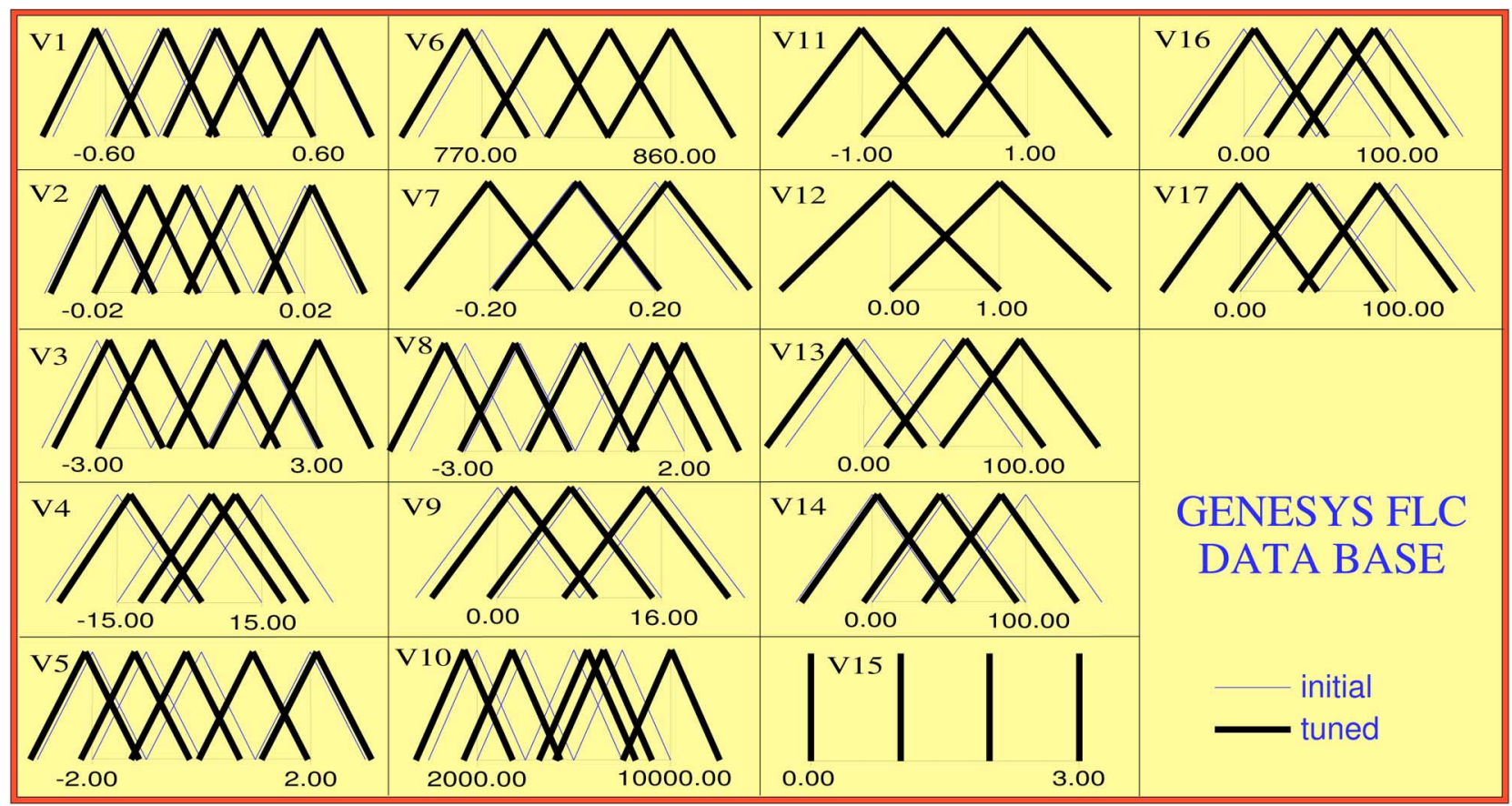

Fig. 16. Initial and tuned $\mathrm{DB}$ obtained by $\mathrm{GL}_{s s}+\mathrm{S}$ (seed 1$)$.

\section{CONCLUSION}

In this paper, a new rule representation scheme using the linguistic 2-tuples representation model has been considered, proposing a new postprocessing method of FRBSs by means of evolutionary lateral tuning of the linguistic variables. Here, we present our conclusions and further considerations.

- The lateral tuning of fuzzy partitions allows an important reduction of the search space from the optimization point of view. This approach works better as the problem search space complexity grows.

- Lateral tuning together with the linguistic 2-tuples representation model offers a good mechanism to obtain an interpretable model, since the shapes of the initial linguistic partitions are preserved and only small displacements are considered.

- The use of rule selection methods to reduce the number of rules while lateral tuning is performed is a good approach to obtain more compact and precise models. This combination increases the search space (tuning of the parameters + selection of the rules), which is easily handled by the simple lateral tuning.

Finally, we should point out that the use of different FRBS learning schemes considering the 2-tuples rule representation model is a good approach to obtain more compact and precise models.

\section{APPENDIX}

\section{The HVAC System FuZZY CONTROL PROBleM}

This Appendix presents a brief introduction to HVAC systems and to their control with FLCs. Then, a real test site and the specific problem considered in this paper are described.

\section{A. HVAC Systems and FLCs}

HVAC systems are equipment usually used to maintain satisfactory conditions of comfort in buildings. An HVAC system is made up of all the components of the appliance used to condition the interior air of a building. The HVAC system is needed to provide the occupants with a comfortable and productive working environment that satisfies their physiological needs. There are no statistical data collected on types and sizes of HVAC systems delivered to each type of building in different European countries. Therefore, providing an HVAC system compatible with the building's environment is a task of the BEMS designer, who depends on experience. In Fig. 18, a typical office-building HVAC system is presented. This system consists of a set of components able to raise and lower the temperature and relative humidity of the supply air.

The energy consumption as well as indoor comfort aspects of ventilated and air conditioned buildings are highly dependent on the design, performance, and control of their HVAC systems and equipments. In a modern intelligent building, a sophisticated control system should provide excellent environmental control [54], [55]. Within this framework (building automation), the objective of a global controller is to maintain the indoor environment within the desired (or stipulated) limits-in our case, to maintain environmental conditions within the comfort zone and to control the indoor air quality. Furthermore, other important objectives are usually required, e.g., energy savings (our main objective), system stability, etc. In any case, numerous factors have to be considered in order to achieve these objectives. This means that the system being controlled is very complex and presents a strong nonlinearity.

Within the framework of machine learning, some artificial intelligence techniques could be successfully applied to enhance the HVAC system capabilities [54]-[62] or to aid the HVAC system modeling [64]. However, most works apply FLCs to 


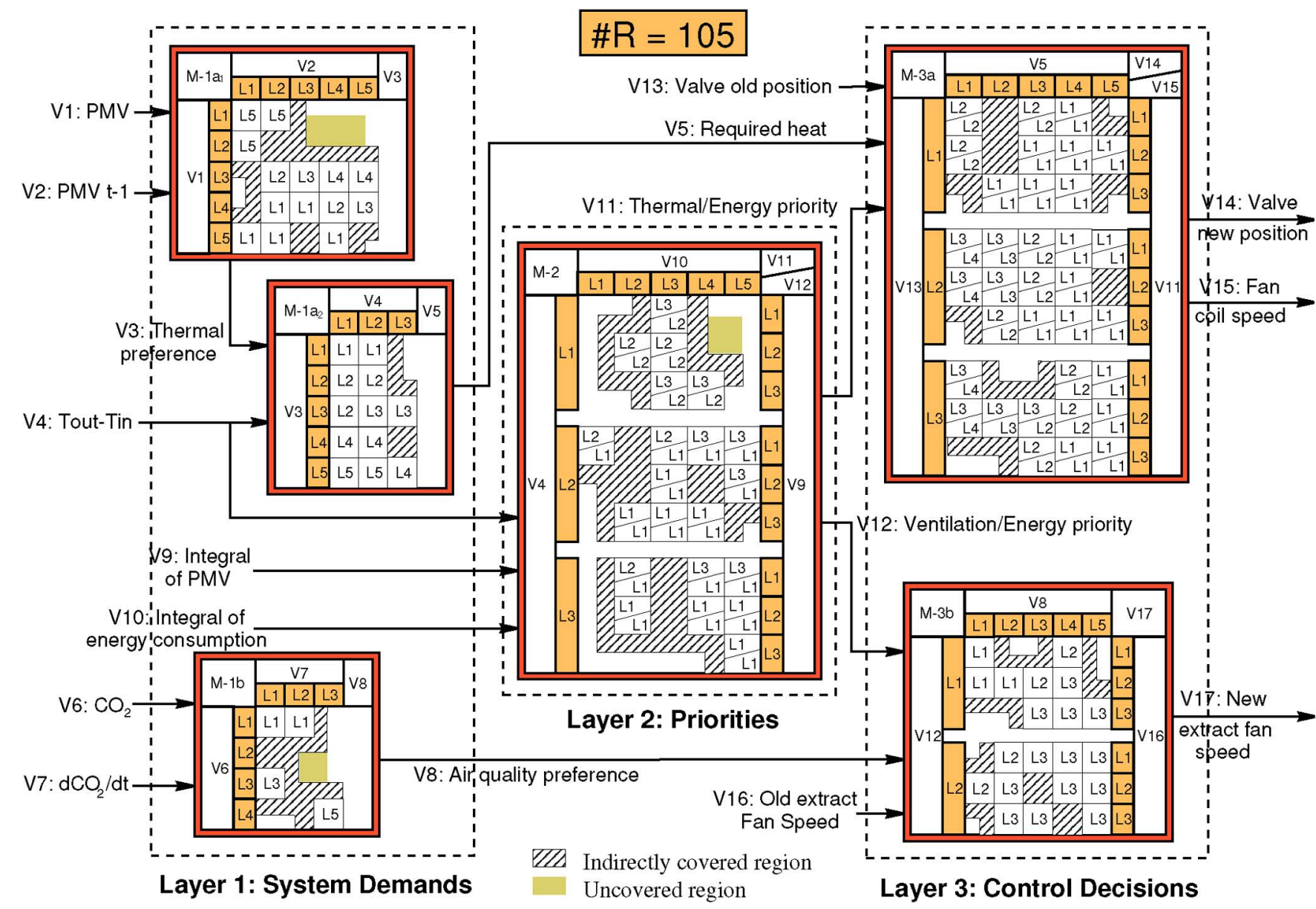

Module $1 \mathrm{a}_{1}$ : Thermal Demands

Module $1 \mathrm{a}_{2}$ : Thermal Preference

Module 1b: Air Quality Demands
Module 2: Energy Priorities

Module 3a: Required HVAC System Status

Module 3b: Required Ventilation System Status

Fig. 17. Final $\mathrm{RB}$ obtained by $\mathrm{GL}_{s s}+\mathrm{S}$ (seed 1).

solve individually simple problems such as thermal regulation (maintaining a temperature setpoint), energy savings, or comfort improvements. On the other hand, the KB is usually constructed based on the operator's control experience, using rules of thumb. However, FLCs sometimes fail to obtain satisfactory results with the initial rule set drawn from the expert's experience [54]. Moreover, since different criteria must be considered, the system being controlled is too complex and optimal FLCs are required. Therefore, this approach needs a modification of the initial KB to obtain an optimal controller with improved performance. In our case, FLCs are obtained from human experience and are subsequently improved by the application of GAs.

\section{B. The Genesys Test Cell}

Within the framework of the GENESYS project, a real test site was provided by a French private enterprise-whose name must remain anonymous. This site will be called the GENESYS test site. Located in France, this test environment consists of seven single zone test cells. Around their walls, an artificial climate can be created at any time. Fig. 19 illustrates this environment. Two adjacent twin cells were available for our experiments, cells number 4 and 5 .
To evaluate the FLC performance, a physical modelization of the controlled buildings and equipments is usually needed. Thus, we will have the chance to evaluate the FLCs designed in the simulated system with the desired environmental conditions. In this way, the first task was to develop the thermal model of this test site. The experimentation of this paper has been performed considering the calibrated and validated GENESYS simulation model, specifically, the GENESYS summer model.

1) Objectives and Fitness Function: Our main optimization objective is energy performance but maintaining the required indoor comfort levels. In this way, the global objective is to minimize the following five criteria.

$\mathbf{O}_{1} \quad$ Upper thermal comfort limit: if PMV $>0.5, O_{1}=O_{1}+(\mathrm{PMV}-0.5)$, where PMV is the more global predicted mean vote thermal comfort index 7730 selected by the International Standard Organization (ISO), incorporating relative humidity and mean radiant temperature. (see http://www.iso.org/iso/en/ISOOnline.frontpage).

$\mathrm{O}_{2} \quad$ Lower thermal comfort limit: if PMV $<-0.5, O_{2}=O_{2}+(-\mathrm{PMV}-0.5)$. 


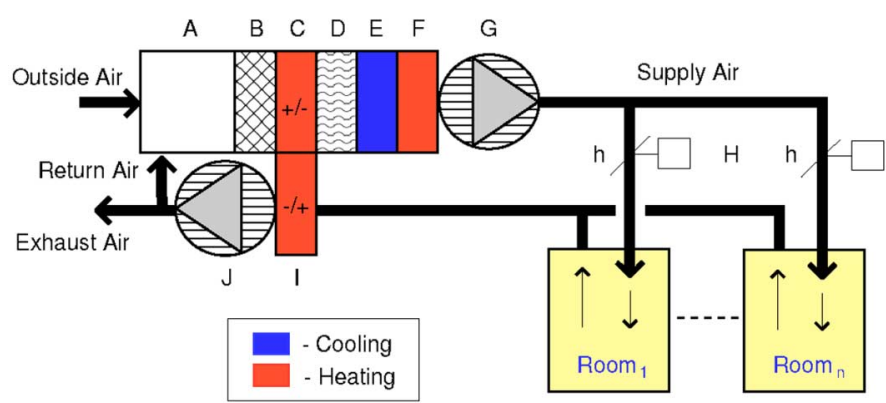

A - This module mixes the return and the outside air to provide supply air, and also closes outside air damper and opens return air damper when fan stops. B - This is a filter to reduce the outside air emissions to supply air. C - The preheater/heat recovery unit preheats the supply air and recovers energy from the exhaust air. D - A humidifier raising the relative humidity in winter. E - This is a cooler to reduce the supply air temperature and/or humidity. F - An after-heater unit to raise the supply air temperature after humidifier or to raise the supply air temperature after latent cooling (dehumidifier). G - The supply air fan. $\mathbf{H}$ - The dampers to demand controlled supply air flow to rooms. I - Heat recovery unit for energy recovery from exhaust air. $\mathbf{J}$ The exhaust air fan.

Fig. 18. Generic structure of an office-building HVAC system.

\section{GENESYS Test Cells}

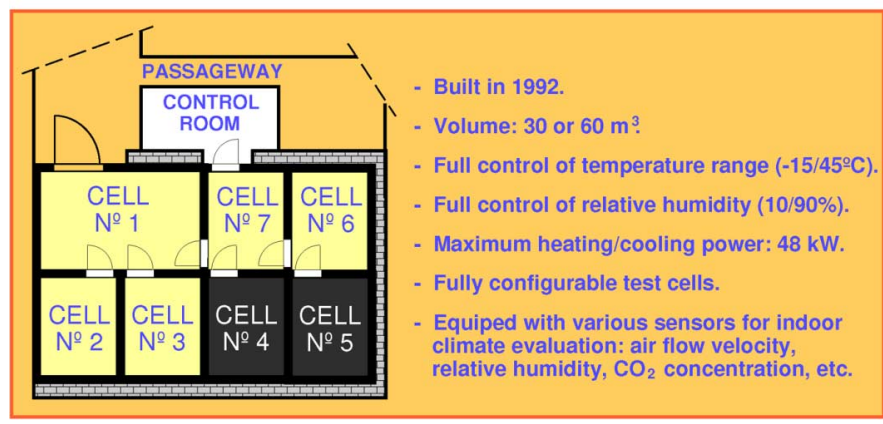

Fig. 19. Representation and main characteristics of the GENESYS test cells.

$\mathrm{O}_{3} \quad$ Indoor air quality requirement: if $\mathrm{CO}_{2}$ conc. $>800$ ppm, $\mathrm{O}_{3}=\mathrm{O}_{3}+\left(\mathrm{CO}_{2}-800\right)$.

$\mathrm{O}_{4} \quad$ Energy consumption: $\mathrm{O}_{4}=\mathrm{O}_{4}+$ Power at time $t$.

$\mathbf{O}_{5} \quad$ System stability: $O_{5}=O_{5}+$ system change from time $t$ to $(t-1)$, where system change states for a change in the system operation, e.g., a change in the fan speed or valve position.

These criteria are combined into one overall objective function by means of a vector of weights. When trustworthy weights are available, this approach reduces the size of the search space providing the adequate direction into the solution space; therefore, its use is highly recommended. In our case, trusted weights were obtained by experts for the objective weighting fitness function: $w_{1}^{O}=0.0083022, w_{2}^{O}=$ $0.0083022, w_{3}^{O}=0.00000456662, w_{4}^{O}=0.0000017832$, and $w_{5}^{O}=0.000761667$. Finally, the fitness function to be minimized could be

$$
F=\sum_{i=1}^{n} w_{i}^{O} \cdot O_{i} .
$$

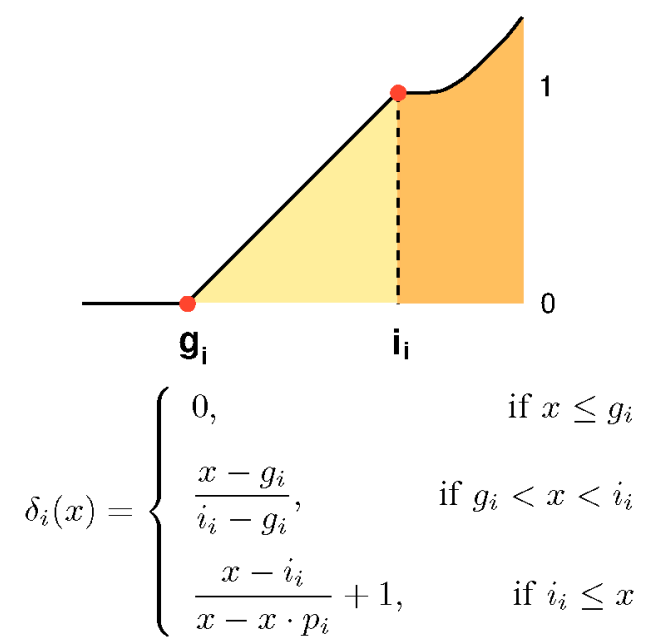

Fig. 20. $\delta_{i}(x)$ when $g_{i} \leq i_{i}$.

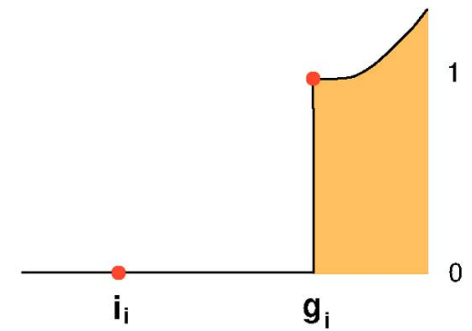

$$
\delta_{i}(x)= \begin{cases}0, & \text { if } x<g_{i} \\ \frac{x-g_{i}}{x-x \cdot p_{i}}+1, & \text { if } g_{i} \leq x\end{cases}
$$

Fig. 21. $\delta_{i}(x)$ when $g_{i}>i_{i}$.

However, the fitness function has been modified in order to consider the use of fuzzy goals that decrease the importance of each individual fitness value whenever it reaches its goal or penalize each objective whenever its value worsens with respect to the initial solution. To do so, a function modifier parameter $\delta_{i}(x)$ (taking values over 1.0) is considered. A penalization rate $p_{i}$ has been included in $\delta_{i}(x)$, allowing the user to set up priorities in the objectives ( 0 less priority and 1 more priority). Therefore, the global fitness is evaluated as

$$
F^{\prime}=\sum_{i=1}^{5} w_{i}^{O} \cdot \delta_{i}\left(O_{i}\right) \cdot O_{i} .
$$

Two situations can be presented according to the value of the goal $g_{i}$ and the value of the initial solution $i_{i}$. Depending on these values, two different $\delta$ functions will be applied.

- When the value of $g_{i}$ is less than the value of $i_{i}$, the objective is not considered if the goal is met and penalized if the initial results are worsened (see Fig. 20).

- When the value of $i_{i}$ is less than the value of $g_{i}$, the initial results can be worsened while the goal is met and is penalized otherwise (see Fig. 21).

2) FLC Variables and Architecture: A hierarchical FLC architecture considering the PMV, $\mathrm{CO}_{2}$ concentration, previous HVAC system status, and outdoor temperature was proposed by 


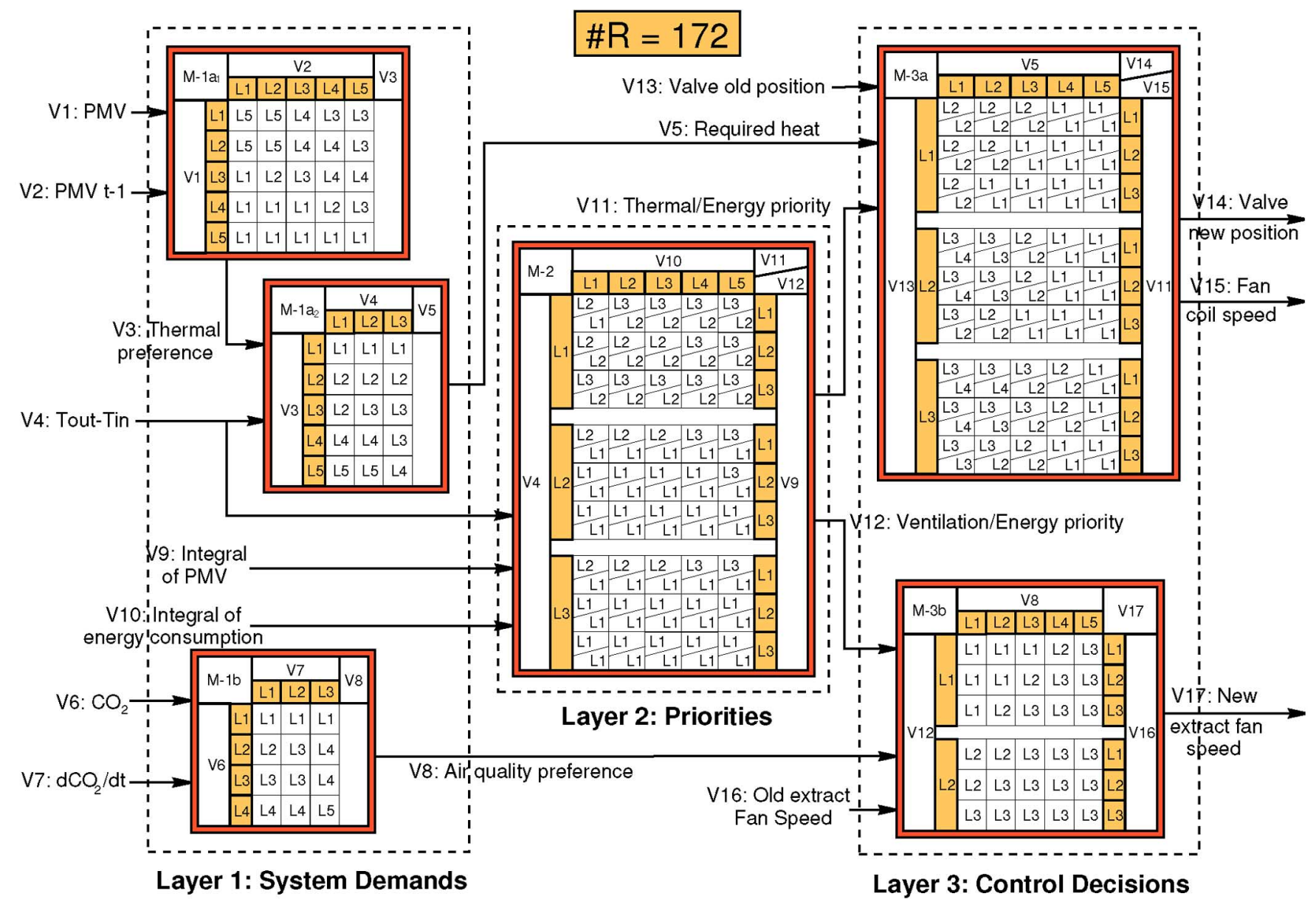
Module $1 a_{1}$ : Thermal Demands
Module $1 \mathrm{a}_{2}$ : Thermal Preference
Module 1b: Air Quality Demands
Module 2: Energy Priorities
Module 3a: Required HVAC System Status
Module 3b: Required Ventilation System Status

Fig. 22. Initial rule base and generic structure of the GENESYS summer FLC.

the BEMS designer for this site. This architecture, variables, and initial RB are presented in Fig. 22.

The initial DB, depicted in Fig. 16 (Section V-B, together with the tuned DB), is composed of symmetrical fuzzy partitions with triangular shaped MFs labeled from $L 1$ to $L l_{i}$ (where $l_{i}$ is the number of labels of the $i$ th variable). Fig. 22 represents the decision tables of each module of the hierarchical FLC in terms of these labels. Each cell of the table represents a fuzzy subspace and contains its associated output consequent(s), i.e., the corresponding label(s). The output variables are shown in the top left square for each module. Both the initial RB and the DB were provided by the BEMS designer.

\section{ACKNOWLEDGMENT}

The authors would like to thank the GENESYS project partners for their careful implementation of the presented HVAC simulation model and for their valuable assistance.

\section{REFERENCES}

[1] L. A. Zadeh, "Fuzzy sets," Inf. Contr., vol. 8, pp. 338-353, 1965.
[2] _ - "Outline of a new approach to the analysis of complex systems and decision processes," IEEE Trans. Syst., Man, Cybern., vol. SMC-3, pp. 28-44, 1973.

[3] - "The concept of a linguistic variable and its applications to approximate reasoning, Parts I, II and III," Inf. Sci., vol. 8, no. 8, 9, pp. 199-249, 301-357, 43-80, 1975.

[4] E. H. Mamdani, "Application of fuzzy algorithms for control of simple dynamic plant," in Proc. Inst. Elect. Eng., 1974, vol. 121, no. 12, pp. $1585-1588$.

[5] E. H. Mamdani and S. Assilian, "An experiment in linguistic synthesis with a fuzzy logic controller," Int. J. Man-Machine Studies, vol. 7, pp. $1-13,1975$.

[6] A. Bastian, "How to handle the flexibility of linguistic variables with applications," Int. J. Uncertainty, Fuzziness Knowledge-Based Syst., vol. 3, no. 4, pp. 463-484, 1994.

[7] J. Casillas, O. Cordón, F. Herrera, and L. Magdalena, Eds., Accuracy Improvements in Linguistic Fuzzy Modeling. Berlin, Germany: Springer-Verlag, 2003.

[8] R. Babuška, J. Oosterhoff, A. Oudshoorn, and P. M. Bruijn, "Fuzzy self-tuning PI control of pH in fermentation," Eng. Applicat. Artif. Intell., vol. 15, no. 1, pp. 3-15, 2002.

[9] O. Cordón, F. Herrera, F. Hoffmann, and L. Magdalena, Genetic Fuzzy Systems: Evolutionary Tuning and Learning of Fuzzy Knowledge Bases, ser. Advances in Fuzzy Systems-Applications and Theory. Singapore: World Scientific, 2001, vol. 19.

[10] F. Herrera, M. Lozano, and J. L. Verdegay, "Tuning fuzzy logic controllers by genetic algorithms," Int. J. Approx. Reason., vol. 12, pp. 299-315, 1995 . 
[11] J. S. R. Jang, "ANFIS: Adaptive network based fuzzy inference system," IEEE Trans. Syst., Man, Cybern., vol. 23, no. 3, pp. 665-684, 1993.

[12] C. Karr, "Genetic algorithms for fuzzy controllers," AI Expert, vol. 6, no. 2, pp. 26-33, 1991.

[13] D. Driankov, H. Hellendoorn, and M. Reinfrank, An Introduction to Fuzzy Control. Berlin, Germany: Springer-Verlag, 1993.

[14] C. Moraga and R. Salas, "A new aspect for the optimization of fuzzy if-then rules," in Proc. 35th Int. Symp. Multiple-Valued Logic (ISMVL'05), 2005, pp. 160-165.

[15] F. Herrera and L. Martínez, "A 2-tuple fuzzy linguistic representation model for computing with words," IEEE Trans. Fuzzy Syst., vol. 8, no. 6, pp. 746-752, 2000.

[16] H. Ishibuchi and T. Yamamoto, "Trade-off between the number of fuzzy rules and their classification performance," in Accuracy Improvements in Linguistic Fuzzy Modeling, J. Casillas, O. Cordón, F. Herrera, and L. Magdalena, Eds. Berlin, Germany: Springer-Verlag, 2003, pp. 72-99.

[17] A. Krone, H. Krause, and T. Slawinski, "A new rule reduction method for finding interpretable and small rule bases in high dimensional search spaces," in Proc. 9th IEEE Int. Conf. Fuzzy Syst., San Antonio, TX, 2000, pp. 693-699.

[18] O. Cordón and F. Herrera, "A three-stage evolutionary process for learning descriptive and approximate fuzzy logic controller knowledge bases from examples," Int. J. Approx. Reason., vol. 17, no. 4, pp. 369-407, 1997.

[19] D. E. Goldberg, Genetic Algorithms in Search, Optimization, and Machine Learning. New York: Addison-Wesley, 1989.

[20] J. H. Holland, Adaptation in Natural and Artificial Systems. Ann Arbor: Univ. of Michigan Press, 1975.

[21] J. Casillas, O. Cordón, F. Herrera, and L. Magdalena, Eds., Interpretability Issues in Fuzzy Modeling. Berlin, Germany: Springer-Verlag, 2003.

[22] J. V. de Oliveira, "Semantic constraints for membership function optimization," IEEE Trans. Syst., Man, Cybern. A, Syst., Humans, vol. 29, no. 1, pp. 128-138, 1999.

[23] — , "Towards neuro-linguistic modeling: Constraints for optimization of membership functions," Fuzzy Sets Syst., vol. 106, no. 3, pp. 357-380, 1999.

[24] U. Bodenhofer and P. Bauer, "A formal model of interpretability of linguistic variables," in Interpretability Issues in Fuzzy Modeling, J. Casillas, O. Cordón, F. Herrera, and L. Magdalena, Eds. Berlin, Germany: Springer-Verlag, 2003, pp. 524-545.

[25] F. Cheong and R. Lai, "Constraining the optimization of a fuzzy logic controller using an enhanced genetic algorithm," IEEE Trans. Syst., Man, Cybern. B, Cybern., vol. 30, no. 1, pp. 31-46, 2000.

[26] J. Espinosa and J. Vandewalle, "Constructing fuzzy models with linguistic integrity from numerical data-AFRELI algorithm," IEEE Trans. Fuzzy Syst., vol. 8, no. 5, pp. 591-600, 2000.

[27] F. Klawonn, "Reducing the number of parameters of a fuzzy system using scaling functions," Soft Comput., vol. 10, no. 9, pp. 749-756, 2006.

[28] O. Cordón, F. Gomide, F. Herrera, F. Hoffmann, and L. Magdalena, "Ten years of genetic fuzzy systems: Current framework and new trends," Fuzzy Sets Syst., vol. 41, no. 1, pp. 5-31, 2004.

[29] L. J. Eshelman, "The CHC adaptive search algorithm: How to have safe search when engaging in nontraditional genetic recombination," in Foundations of Genetic Algorithms, G. Rawlin, Ed. San Mateo, CA: Morgan Kaufman, 1991, vol. 1, pp. 265-283.

[30] D. Whitley and J. Kauth, "GENITOR: A different genetic algorithm," in Proc. Rocky Mountain Conf. Artif. Intell., Denver, 1988, pp. $118-130$.

[31] F. Herrera, M. Lozano, and A. M. Sánchez, "A taxonomy for the crossover operator for real-coded genetic algorithms: An experimental study," Int. J. Intell. Syst., vol. 18, pp. 309-338, 2003.

[32] F. Herrera, M. Lozano, and J. L. Verdegay, "Fuzzy connectives based crossover operators to model genetic algorithms population diversity," Fuzzy Sets Syst., vol. 92, no. 1, pp. 21-30, 1997.

[33] L. X. Wang and J. M. Mendel, "Generating fuzzy rules by learning from examples," IEEE Trans. Syst., Man, Cybern., vol. 22, no. 6, pp. $1414-1427,1992$.

[34] K. Nozaki, H. Ishibuchi, and H. Tanaka, "A simple but powerful heuristic method for generating fuzzy rules from numerical data," Fuzzy Sets Syst., vol. 86, no. 3, pp. 251-270, 1997.

[35] P. Carmona, J. Castro, and J. Zurita, "Strategies to identify fuzzy rules directly from certainty degrees: A comparison and a proposal," IEEE Trans. Fuzzy Syst., vol. 12, no. 5, pp. 631-640, 2004.
[36] O. Cordón and F. Herrera, "A proposal for improving the accuracy of linguistic modeling," IEEE Trans. Fuzzy Syst., vol. 8, no. 3, pp. 335-344, 2000.

[37] H. Ishibuchi, T. Murata, and I. B. Türksen, "Single-objective and twoobjective genetic algorithms for selecting linguistic rules for pattern classification problems," Fuzzy Sets Syst., vol. 89, no. 2, pp. 135-150, 1997.

[38] F. Herrera, M. Lozano, and J. L. Verdegay, "A learning process for fuzzy control rules using genetic algorithms," Fuzzy Sets Syst., vol. 100, pp. 143-158, 1998.

[39] H. Ishibuchi, K. Nozaki, N. Yamamoto, and H. Tanaka, "Selecting fuzzy if-then rules for classification problems using genetic algorithms," IEEE Trans. Fuzzy Syst., vol. 3, no. 3, pp. 260-270, 1995.

[40] A. Krone and H. Taeger, "Data-based fuzzy rule test for fuzzy modelling," Fuzzy Sets Syst., vol. 123, no. 3, pp. 343-358, 2001.

[41] Y. Yam, P. Baranyi, and C. T. Yang, "Reduction of fuzzy rule base via singular value decomposition," IEEE Trans. Fuzzy Syst., vol. 7, pp. 120-132, 1999.

[42] J. Yen and L. Wang, "Simplifying fuzzy rule-based models using orthogonal transformation methods," IEEE Trans. Syst., Man, Cybern. B, Cybern., vol. 29, pp. 13-24, 1999.

[43] A. Klose, A. Nurnberger, and D. Nauck, "Some approaches to improve the interpretability of neuro-fuzzy classifiers," in Proc. 6th Eur. Congr. Intell. Techn. Soft Comput., Aachen, Germany, 1998, pp. 629-633.

[44] T. P. Hong and C. Y. Lee, "Effect of merging order on performance of fuzzy induction," Intell. Data Anal., vol. 3, no. 2, pp. 139-151, 1999.

[45] M. Setnes, R. Babuška, U. Kaymak, and H. R. van Nauta Lemke, "Similarity measures in fuzzy rule base simplification," IEEE Trans. Syst., Man, Cybern. B, Cybern., vol. 28, no. 3, pp. 376-386, 1998.

[46] M. Setnes and J. A. Roubos, "GA-fuzzy modeling and classification: Complexity and performance," IEEE Trans. Fuzzy Syst., vol. 8, no. 5, pp. 509-522, 2000.

[47] J. A. Roubos and M. Setnes, "Compact and transparent fuzzy models and classifiers through iterative complexity reduction," IEEE Trans. Fuzzy Syst., vol. 9, no. 4, pp. 516-524, 2001.

[48] A. F. Gómez-Skarmeta and F. Jiménez, "Fuzzy modeling with hybrid systems," Fuzzy Sets Syst., vol. 104, pp. 199-208, 1999.

[49] J. Casillas, O. Cordón, M. J. D. Jesus, and F. Herrera, "Genetic tuning of fuzzy rule deep structures preserving interpretability and its interaction with fuzzy rule set reduction," IEEE Trans. Fuzzy Syst., vol. 13, no. 1, pp. 13-29, 2005.

[50] R. Alcalá, J. Alcalá-Fdez, J. Casillas, O. Cordón, and F. Herrera, "Hybrid learning models to get the interpretability-accuracy trade-off in fuzzy modeling," Soft Comput., vol. 10, no. 9, pp. 717-734, 2006.

[51] O. Cordón, F. Herrera, and L. Sánchez, "Solving electrical distribution problems using hybrid evolutionary data analysis techniques," Appl. Intell., vol. 10, pp. 5-24, 1999.

[52] R. Alcalá, J. M. Benítez, J. Casillas, O. Cordón, and R. Pérez, "Fuzzy control of HVAC systems optimized by genetic algorithms," Appl. Intell., vol. 18, pp. 155-177, 2003.

[53] A. L. Dexter, D. Phil, and C. Eng, "Intelligent buildings: Fact or fiction?," HVAC \& R Res., vol. 2, no. 2, pp. 105-123, 1996.

[54] S. Huang and R. M. Nelson, "Rule development and adjustment strategies of a fuzzy logic controller for an HVAC system-Parts I and II (analysis and experiment)," ASHRAE Trans., vol. 100, no. 1, pp. 841-850, 851-856, 1994.

[55] M. Arima, E. H. Hara, and J. D. Katzberg, "A fuzzy logic and rough sets controller for HVAC systems," in Proc. IEEE WESCANEX'95, New York, 1995, vol. 1, pp. 133-138.

[56] F. Calvino, M. L. Gennusa, G. Rizzo, and G. Scaccianoce, "The control of indoor thermal comfort conditions: Introducing a fuzzy adaptive controller," Energy Build., vol. 36, pp. 97-102, 2004.

[57] P. Y. Glorennec, "Application of fuzzy control for building energy management," in Building Simulation: International Building Performance Simulation Association. Paris, France: Sophia Antipolis, 1991, vol. 1, pp. 197-201.

[58] L. Lu, W. Cai, L. Xie, S. Li, and Y.C. Soh, "HVAC system optimization in building section," Energy Build., vol. 37, pp. 11-22, 2005.

[59] J. Pargfrieder and H. Jörgl, "An integrated control system for optimizing the energy consumption and user comfort in buildings," in Proc. 12th IEEE Int. Symp. Comput. Aided Contr. Syst. Design, Glasgow, Scotland, U.K., 2002, pp. 127-132.

[60] A. Rahmati, F. Rashidi, and M. Rashidi, "A hybrid fuzzy logic and PID controller for control of nonlinear HVAC systems," in Proc. IEEE Int. Conf. Syst., Man, Cybern, Washington, DC, 2003, vol. 3, pp. 2249-2254. 
[61] J. Wu and W. Cai, "Development of an adaptive neuro-fuzzy method for supply air pressure control in HVAC system," in Proc. IEEE Int. Conf. Syst., Man, Cybern., Nashville, TN, 2000, vol. 5, pp. 3806-3809.

[62] I. H. Yang, M. S. Yeo, and K. W. Kim, "Application of artificial neural network to predict the optimal start time for heating system in building," Energy Conv. Manage., vol. 44, pp. 2791-2809, 2003.

[63] R. Alcalá, J. Casillas, O. Cordón, A. González, and F. Herrera, “A genetic rule weighting and selection process for fuzzy control of heating, ventilating and air conditioning systems," Eng. Applicat. Artif. Intell., vol. 18, no. 3, pp. 279-296, 2005.

[64] P. P. Angelov, Evolving Rule-Based Models: A Tool for Design of Flexible Adaptive Systems, ser. Studies in Fuzziness and Soft Computing. Berlin, Germany: Physica-Verlag, 2002, vol. 92.

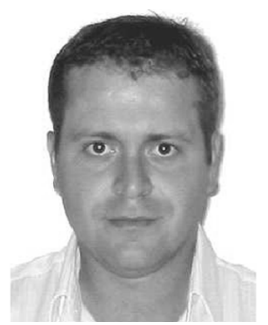

Rafael Alcalá received the M.Sc. and Ph.D. degrees in computer science from the University of Granada, Spain, in 1998 and 2003, respectively.

$\mathrm{He}$ is currently an Assistant Professor in the Department of Computer Science and Artificial Intelligence, University of Granada, where he is a Member of the Soft Computing and Intelligent Information Systems Research Group. He has more than 37 international publications, 12 of them published in international journals. He has worked on several research projects supported by the Spanish government and the European Union. His research interests include multiobjective genetic algorithms and genetic fuzzy systems, especially the learning/tuning of fuzzy systems for modeling and control with tradeoff between interpretability and accuracy.

Prof. Alcalá coedited the IEEE TRANSACTIONS ON FuZZY Systems Special Issue on "Genetic Fuzzy Systems: What's Next."

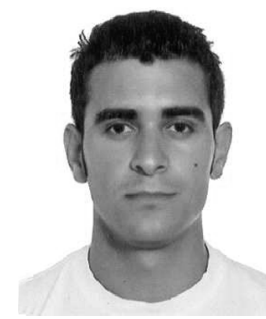

Jesús Alcalá-Fdez was born in Córdoba, Spain, in 1979. He received the M.Sc. degree in computer science from the University of Granada, Spain, in 2002.

$\mathrm{He}$ is an Assistant Professor in the Department of Computer Science, University of Jaén, Spain. He is a Member of the Soft Computing and Intelligent Information Systems Research Group, Department of Computer Science and Artificial Intelligence, University of Granada. He has more than 15 international publications, six of them published in international journals. His research interests include genetic fuzzy systems, especially the learning/tuning of fuzzy systems for modeling and control with tradeoff between interpretability and accuracy.

Prof. Alcalá-Fdez coedited the IEEE TRANSACTIONS ON FuZZY Systems Special Issue on "Genetic Fuzzy Systems: What's Next."

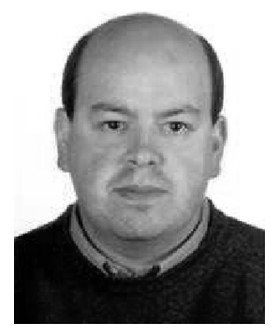

Francisco Herrera received the M.Sc. and Ph.D. degrees in mathematics from the University of Granada, Spain, in 1988 and 1991, respectively.

$\mathrm{He}$ is currently a Professor in the Department of Computer Science and Artificial Intelligence, University of Granada. He has published more than 100 papers in international journals. He is coauthor of Genetic Fuzzy Systems: Evolutionary Tuning and Learning of Fuzzy Knowledge Bases (Singapore: World Scientific, 2001). He has coedited three international books and 15 special issues in international journals on different soft computing topics, such as, "Preference Modelling," "Computing with Words," "Genetic Algorithms," and "Genetic Fuzzy Systems." He currently serves on the editorial boards of Soft Computing, Mathware and Soft Computing, International Journal of Hybrid Intelligent Systems, International Journal of Computational Intelligence Research, International Journal of Information Technology and Intelligent Computing, and Evolutionary Computation. His current research interests include computing with words, preference modelling and decision making, data mining and knowledge discovery, genetic algorithms, and genetic fuzzy systems. 